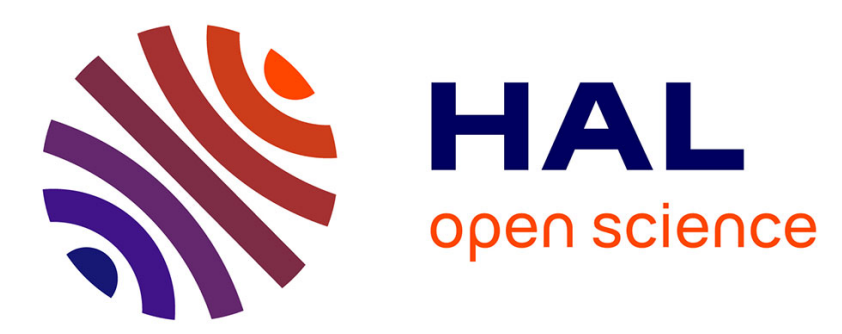

\title{
The injectivity of the canonical signal module for multidimensional linear systems of difference equations with variable coefficients
}

Henri Bourlès, Bogdan Marinescu, U Oberst

\section{- To cite this version:}

Henri Bourlès, Bogdan Marinescu, U Oberst. The injectivity of the canonical signal module for multidimensional linear systems of difference equations with variable coefficients. Multidimensional Systems and Signal Processing, 2015, 10.1007/s110451-015-0331-x . hal-02368215v2

HAL Id: hal-02368215

https://hal.science/hal-02368215v2

Submitted on 22 Dec 2016

HAL is a multi-disciplinary open access archive for the deposit and dissemination of scientific research documents, whether they are published or not. The documents may come from teaching and research institutions in France or abroad, or from public or private research centers.
L'archive ouverte pluridisciplinaire HAL, est destinée au dépôt et à la diffusion de documents scientifiques de niveau recherche, publiés ou non, émanant des établissements d'enseignement et de recherche français ou étrangers, des laboratoires publics ou privés. 


\title{
The injectivity of the canonical signal module for multidimensional linear systems of difference equations with variable coefficients
}

\author{
dedicated to Professor Ettore Fornasini on the occasion of his seventieth birthday \\ published in Multidimensional Systems and Signal Processing 2015
}

DOI 10.1007/s110451-015-0331-x

H. Bourlès $\stackrel{*}{,}$ B. Marinescu ${ }^{\dagger}$ U. Oberst ${ }^{\ddagger}$

May 17, 2015

\begin{abstract}
We consider discrete behaviors with varying coefficients. Our results are new also for one-dimensional systems over the time-axis of natural numbers and for varying coefficients in a field, we derive the results, however, in much greater generality: Instead of the natural numbers we use an arbitrary submonoid $\mathrm{N}$ of an abelian group, for instance the standard multidimensional lattice of $r$-dimensional vectors of natural numbers or integers. We replace the base field by any commutative self-injective ring $\mathrm{F}$, for instance a direct product of fields or a quasi-Frobenius ring or a finite factor ring of the integers. The F-module $\mathrm{W}$ of functions from $\mathrm{N}$ to $\mathrm{F}$ is the canonical discrete signal module and is a left module over the natural associated noncommutative ring A of difference operators with variable coefficients. Our main result states that this module is injective and therefore satisfies the fundamental principle: An inhomogeneous system of linear difference equations with variable coefficients has a solution if and only if the right side satisfies the canonical compatibility conditions. We also show that for the typical cases of partial difference equations and in contrast to the case of constant coefficients the A-module $\mathrm{W}$ is not a cogenerator. We also generalize the standard one-dimensional theory for periodic coefficients to the multidimensional situation by invoking Morita equivalence.
\end{abstract}

AMS-classification: 93C05, 93C55, 16D50, 39A06, 39A14

Key-words: difference equation, variable coefficient, injective signal module, fundamental principle, cogenerator, periodic

*SATIE, ENS Cachan/CNAM, 61 Avenue President Wilson, F-94230, Cachan, France. email: henri.bourles@satie.ens-cachan.fr

†École Centrale de Nantes, B.P. 92101, 1, rue de la Noë, FR-44321 Nantes Cedex 3, France. email: bogdan.marinescu@irccyn.ec-nantes.fr

${ }^{\ddagger}$ Institut für Mathematik, Universität Innsbruck, Technikerstrasse 13, A-6020 Innsbruck, Austria. email: ulrich.oberst@uibk.ac.at 


\section{Introduction}

Most papers on discrete linear time-varying (LTV) systems theory discuss Kalman's state space systems over a base field $\mathbb{F}$; compare Rugh's excellent book [21, pp.383568]. The existence of (unique) states and output for given input and initial state are obvious for these systems. In this paper we consider arbitrary discrete LTV-behaviors. Our results are new also for the time-axis $\mathbb{N}$ of natural numbers and varying coefficients in a field, we prove the results, however, in much greater generality: The time axis $\mathbb{N}$ is replaced by any submonoid $N$ of an abelian group, for instance by the $r$-dimensional lattices $N:=\mathbb{N}^{r_{1}} \times \mathbb{Z}^{r_{2}}, r:=r_{1}+r_{2}$, of integers. Instead of a base field we employ an arbitrary commutative self-injective ring $\mathbb{F}$, for instance a direct product of fields or a quasi-Frobenius ring or a finite factor ring $\mathbb{Z} / \mathbb{Z} n$ of $\mathbb{Z}$. We consider the $\mathbb{F}$-module $W:=\mathbb{F}^{N}$ of functions from $N$ to $\mathbb{F}$ as the canonical discrete signal module over the natural associated noncommutative ring $\mathbf{A}$ of difference operators with variable coefficients. Our main result Thm. 2.1 states that $W$ is an injective A-left module and therefore also satisfies the fundamental principle (terminology of Ehrenpreis 1960): An inhomogeneous system of linear difference equations with variable coefficients has a solution if and only if the right side satisfies the canonical compatibility conditions. We also show in Thm. 2.3 that for the standard partial difference equations and in contrast to the case of constant coefficients the canonical signal module ${ }_{\mathbf{A}} W$ is not $a$ cogenerator, cf. (22). This signifies that the equations of a homogeneous system of linear partial difference equations with variable coefficients contain more information than the module of solutions of the system. In Section 4 we generalize previous work of many colleagues on one-dimensional discrete LTV-behaviors with periodic coefficients in a field, cf. for instance, [12], [1], [2], to multidimensional behaviors on the lattice $N=\mathbb{Z}^{r}$ with periodic coefficients in a quasi-Frobenius base ring $\mathbb{F}$ and to the associated ring $\mathbf{A}_{\text {per }}$ of partial difference operators with periodic coefficients. Via Morita equivalence we show in Thms. 4.4, 4.6, 4.7 that $\mathbf{A}_{\text {per }} \mathbb{F}^{N}$ is an injective cogenerator and that every $\mathbf{A}_{\text {per }} \mathbb{F}^{N}$-behavior is isomorphic to its stacked form that is a standard multidimensional behavior over a multivariate Laurent polynomial algebra. In Section 4.4 we also consider the case of periodic trajectories, cf. [23].

The Sections 3.1, 3.2 resp. 4 serve the proof of Thms. $2.1,2.3$ resp. 4.4, 4.6, 4.7. In Section 5 that was added in the revised version of this paper we explain the connection of the multidimensional theory of Section 4 with the previous work in [12], [1], [2].

Remark 1.1. (i) History: These remarks concern the history of the module-behavior duality. The standard operator algebra for linear systems of ordinary or partial difference or differential equations with constant coefficients in $r$ independent variables is the commutative polynomial algebra $\mathbf{A}=\mathbb{F}\left[s_{1}, \cdots, s_{r}\right]$ over a field $\mathbb{F}$ that acts on the canonical signal spaces $W$ of $r$-variate sequences resp. smooth functions or distributions via partial shifts resp. differentiation. In [18] it was shown that these signal modules are injective cogenerators, cf. also (20), and thus give rise to a categorical duality or very strong one-one correspondence between finitely generated (f.g.) Amodules and the solution spaces of these systems (terminology of Malgrange 1962), called behaviors by Willems. The proof of the injectivity for partial differential equations was outstanding analytic work by Ehrenpreis, Malgrange and Palamodov in the 1960s. The duality enables the translation of theorems and algorithms from Commutative Algebra and Homological Algebra into analytic and systems theoretic results. This procedure was and is called Algebraic Analysis, in particular by Pommaret and his student and coauthor Quadrat [19] and by other colleagues [20] from Systems The- 
ory and Computer Algebra. In 1959 Sato introduced the term Algebraic Analysis for the advanced algebraic theory of partial differential equations whose most significant progress is due to Kashiwara [11], but it seems that the word was already used by Euler. The duality theory with its translation of algebra into systems theory has been used implicitly, but significantly already in Kalman's state space, Rosenbrock's polynomial matrix, Wolovich's differential operator and Willems' behavioral approach to LTI (linear time-invariant)-systems theory.

For systems of equations with variable coefficients the associated rings $\mathbf{A}$ of operators are noncommutative and the relevant signal spaces are, in general, neither injective nor cogenerators, an exception is established in [9]. In many cases the operator ring A is a noetherian domain or, more specifically, an Ore algebra or multivariate skewpolynomial algebra [20, Def. 3.4]. There is a large body of results and algorithms on f.g. A-modules in the theory of Noncommutative Noetherian Rings and Computer Algebra [16], [14], [7], [20], [19] that can, however, be only partially translated into systems theoretic results and algorithms [20], [19]. The term Algebraic Analysis and the suggestive systems theoretic terminology for purely algebraic objects are also used in this more general situation. For instance, a f.g. module resp. some of its distinguished elements are called a system resp. its inputs and outputs [8]. But one has always to keep in mind that the translation of those strong algebraic results into the behavior of actual signals is not obvious if the signal module is not injective. Rings of delay-differential or convolution operators are commutative domains, but not noetherian, and the module-behavior duality also holds only partially [10], [20], [6]. In the present paper the algebra $\mathbf{A}$ of difference operators is neither a domain nor noetherian and it is therefore rather surprising that the canonical signal module is injective.

(ii) Constructivity: The injectivity, even of a field as module over itself, is not a constructive property since, for instance, the Hahn-Banach theorem for infinite-dimensional vector spaces requires the nonconstructive axiom of choice or Zorn's Lemma. Therefore our main Thm. 2.1 is not constructive in general, even in the simplest case of Ex. 2.2 below. Since the ring $\mathbf{A}$ is not noetherian it is, in general, also impossible to compute the canonical compatibility conditions. To construct at least approximate solutions the theory of this paper has to be extended to topological rings of sequences, for instances the Banach algebras $\ell^{1}(\mathbb{Z}), \ell^{\infty}(\mathbb{Z}) \subset \mathbb{C}^{\mathbb{Z}}$. All results on periodic multidimensional behaviors over constructive quasi-Frobenius rings like $\mathbb{Z} / \mathbb{Z} n$ are, however, constructive and can be used with existing Computer Algebra packages, for instance [7], [20], [19]. We leave it to these or other experts to actually do this.

Acknowledgement: We thank the two reviewers for their careful reading of and useful suggestions for the paper.

\section{Basic notions and principal results}

Instead of a field we use a commutative base ring $\mathbb{F}$ that is self-injective or, equivalently, for which an analogue of the Hahn-Banach theorem holds. The ring $\mathbb{F}$ is called self-injective resp. a cogenerator ring if it is injective resp. a cogenerator as module over itself (cf. (9) and (22)). If $\mathbb{F}$ is a cogenerator ring then it is also self-injective [14, Thm. 19.18]. A noetherian (commutative) cogenerator ring is called a (commutative) quasi-Frobenius ring and is artinian [14, Thm. 15.1, §15D]. For any Dedekind domain $D$, especially for any principal ideal domain like $\mathbb{Z}$ and any nonzero ideal $\mathfrak{b} \subsetneq D$ the factor ring $D / \mathfrak{b}$ is a quasi-Frobenius ring [14, Example 15.26, (2)]. An infinite product 
of fields, especially an infinite power, is self-injective, but not a cogenerator ring [14, $\S 19$, Exercise 17 on p.539].

Linear difference equations with constant coefficients over commutative quasi-Frobenius base rings $\mathbb{F}$ were first studied by Russian coding theorists, in particular by Kurakin, Kuzmin, Mikhalev, Nechaev [17], and later in behavioral systems theory, for instance by Liu, Lu, Oberst [15], Kuijper, Pinto, Polderman, Rocha [13], and in several papers by Zerz and her cooperator Wagner [23],[24].

Let $N$ be a submonoid of an abelian group, i.e., $0 \in N$ and $N+N=N$, and $Z:=N-N \supseteq N$ the subgroup generated by $N$. The standard examples for this situation are

$$
Z:=\mathbb{Z}^{r}, r \in \mathbb{N}, \text { and } N:=\mathbb{N}^{r_{1}} \times \mathbb{Z}^{r_{2}} \subset \mathbb{Z}^{r}=\mathbb{Z}^{r_{1}} \times \mathbb{Z}^{r_{2}}, r=r_{1}+r_{2} .
$$

Consider the standard signal $\mathbb{F}$-module for discrete systems theory

$$
W:=\mathbb{F}^{N}:=\left\{w=(w(t))_{t \in N}: N \rightarrow \mathbb{F}, t \mapsto w(t)\right\}
$$

of functions from $N$ to $\mathbb{F}$. For $N:=\mathbb{N}^{r_{1}} \times \mathbb{Z}^{r_{2}}$ from (1) $\mathbb{F}^{N}$ consists of the typical multivariate sequences. As usual the $\mathbb{F}$-module of $k \times \ell$-matrices is denoted by $\mathbb{F}^{k \times \ell}$, in particular we obtain the modules $\mathbb{F}^{1 \times \ell}$ resp. $\mathbb{F}^{\ell}:=\mathbb{F}^{\ell \times 1}$ of row resp. column vectors. We identify

$$
\begin{gathered}
\left(\mathbb{F}^{k \times \ell}\right)^{N}=\left(\mathbb{F}^{N}\right)^{k \times \ell} \ni X=(X(t))_{t \in N}=\left(X_{i j}\right)_{i \leq k, j \leq \ell}, \\
X_{i j} \in \mathbb{F}^{N}, X(t) \in \mathbb{F}^{k \times \ell}, X_{i j}(t)=X(t)_{i j} .
\end{gathered}
$$

The module $\mathbb{F}^{N}$ is an $\mathbb{F}$-algebra with the componentwise multiplication $(a b)(t):=$ $a(t) b(t)$ for $a, b \in \mathbb{F}^{N}$ and the one-element $(1)_{t \in N}=(t \mapsto 1)$. Moreover $N$ acts on $\mathbb{F}^{N}$ by algebra endomorphisms via

$$
(i \circ a)(t)=a(t+i), a \in \mathbb{F}^{N}, i, t \in N .
$$

The module $\mathbb{F}^{N}$ with this additional structure is denoted by $\mathbf{K}$. It gives rise to the noncommutative skew-monoid algebra or smash product [16, Thm. 1.5.4]

$$
\begin{aligned}
& \mathbf{A}:=N \sharp \mathbf{K}:=\mathbf{K}[N ; \circ]=\oplus_{i \in N} \mathbf{K} q^{i} \ni f=\sum_{i \in N} f_{i} q^{i}, f_{i} \in \mathbf{K}, \\
& \text { with the multiplication } \\
&\left(\sum_{i_{1} \in N} f_{i_{1}} q^{i_{1}}\right)\left(\sum_{i_{2} \in N} f_{i_{2}} q^{i_{2}}\right):=\sum_{i \in N}\left(\sum_{i_{1}, i_{2} \in N, i_{1}+i_{2}=i} f_{i_{1}}\left(i_{1} \circ f_{i_{2}}\right)\right) q^{i} .
\end{aligned}
$$

By definition the family $\left(q^{i}\right)_{i \in N}$ is a $\mathbf{K}$-basis of $\mathbf{A}$ with the multiplication $q^{i} q^{j}=$ $q^{i+j}$. Its introduction serves to transform the additive monoid $N$ into the multiplicative monoid $\left\{q^{i} ; i \in N\right\}$. In $f=\sum_{i \in N} f_{i} q^{i} \in \mathbf{A}$ almost all coefficients $f_{i}$, i.e., up to finitely many, are zero by definition. If

$$
\begin{gathered}
N=\mathbb{N}^{r}=\oplus_{\rho=1}^{r} \mathbb{N} \epsilon_{\rho}, \epsilon_{\rho}:=(0, \cdots, 0, \stackrel{\rho}{1}, 0, \cdots, 0), \rho=1, \cdots, r, x_{\rho}:=q^{\epsilon_{\rho}} \\
\text { then } \forall i=\left(i_{1}, \cdots, i_{r}\right) \in \mathbb{N}^{r}: q^{i}=x_{1}^{i_{1}} * \cdots * x_{r}^{i_{r}}=: x^{i}
\end{gathered}
$$

is the usual power product of the $x_{1}, \cdots, x_{r}$ and this motivates the notation $q^{i}$ also for general $N$. For $N \neq 0$ the rings $\mathbb{F}^{N}$ and $\mathbf{A}$ have zero-divisors. For nonnoetherian 
$\mathbb{F}$ or infinite $N$ neither $\mathbf{K}$ nor $\mathbf{A}$ are noetherian. Let ${ }_{F}$ Mod resp. A Mod denote the categories of $\mathbb{F}$ - resp. of left $\mathbf{A}$-modules. Little is known about ${ }_{\mathbf{A}} \mathbf{M o d}$ whereas there are some important results on ${ }_{\mathbb{F}} \mathbf{M o d}\left[14\right.$, Chs. 6,7]. The algebra $\mathbf{A}$ acts on $W=\mathbb{F}^{N}$ via the action $\circ$ given by

$$
\left(\left(\sum_{i \in N} f_{i} q^{i}\right) \circ w\right)(t):=\sum_{i \in N} f_{i}(t) w(t+i),\left(q^{i} \circ w\right)(t)=w(t+i), t, i \in N
$$

and makes $W$ an A-left module, denoted by $\mathbf{A} W$. The action is extended to the action $R \circ w$ of a matrix $R \in \mathbf{A}^{k \times \ell}$ on $w \in W^{\ell}$ by

$$
\begin{gathered}
R=\left(R_{\mu \nu}\right)_{1 \leq \mu \leq k, 1 \leq \nu \leq \ell}=\sum_{j \in N} R_{j} q^{j} \in \mathbf{A}^{k \times \ell}, R_{\mu \nu}=\sum_{j \in N} R_{\mu \nu j} q^{j} \in \mathbf{A}, R_{\mu \nu j} \in \mathbf{K} \\
R_{j}=\left(R_{\mu \nu j}\right)_{1 \leq \mu \leq k, 1 \leq \nu \leq \ell} \in \mathbf{K}^{k \times \ell}, R \circ w:=\left(\sum_{\nu=1}^{\ell} R_{\mu \nu} \circ w_{\nu}\right)_{\mu=1, \cdots, k} \in W^{k}, \\
\forall t \in N:(R \circ w)(t)=\sum_{j \in N} R_{j}(t) w(t+j) \in \mathbb{F}^{k} .
\end{gathered}
$$

Theorem 2.1. For the signal module ${ }_{\mathbf{A}} W$ from (2) and (7) the following hold: (1)The A-module $W$ is injective, i.e., the functor

$$
\operatorname{Hom}_{\mathbf{A}}(-, W): \mathbf{A}_{\mathbf{A}} \mathbf{M o d} \rightarrow{ }_{\mathbb{F}} \operatorname{Mod}, M \mapsto \operatorname{Hom}_{\mathbf{A}}(M, W),
$$

is exact or, equivalently, transforms injective A-linear maps into surjective $\mathbb{F}$-linear maps.

(2) This implies that ${ }_{\mathbf{A}} W$ also satisfies the fundamental principle (terminology of Ehrenpreis 1960): For $R \in \mathbf{A}^{k \times \ell}$ and $u \in W^{k}$ the inhomogeneous linear system

$$
R \circ w=u \text { or } \forall t \in N: \sum_{j \in N} R_{j}(t) w(t+j)=u(t)
$$

has a solution $w \in W^{\ell}$ if and only if the given right side $u$ satisfies the canonical compatibility conditions

$$
\forall \eta \in \mathbf{A}^{1 \times k}: \eta R=0 \Longrightarrow \eta \circ u=0 .
$$

This also holds if $R$ is a row-finite matrix with infinitely many rows and columns, $c f$. Cor. 3.1.

The necessity of (11) for the existence of a solution $w$ of $R \circ w=u$ follows from

$$
\eta R=0 \Longrightarrow \eta \circ u=\eta \circ(R \circ w)=(\eta R) \circ w=0 \circ w=0 .
$$

For $N=\mathbb{N}^{r_{1}} \times \mathbb{Z}^{r_{2}}$ (10) is the typical $r_{1}+r_{2}$-dimensional linear system of partial difference equations with variable coefficients.

Example 2.2. In Thm. 2.1 consider the simple data

$$
\begin{gathered}
N:=\mathbb{N}, \text { a field } \mathbb{F}, \mathbf{A}=\mathbb{F}^{\mathbb{N}}[q ; \circ], W:=\mathbb{F}^{\mathbb{N}}, k=\ell=1, \\
R:=f=f_{d} q^{d}+\cdots+f_{0} \in \mathbf{A}, f_{i} \in \mathbb{F}^{\mathbb{N}}, 0 \neq f_{d}, u \in W .
\end{gathered}
$$


Then there is a solution $w \in W$ of

$$
\begin{gathered}
f \circ w=u \text { or } \forall t \in \mathbb{N}: f_{d}(t) w(t+d)+\cdots+f_{0}(t) w(t)=u(t) \text { if and only if } \\
\forall g=\sum_{j=0}^{\infty} g_{j} q^{j} \in \mathbf{A} \text { with } g f=0: g \circ u=0 \text { or } \forall t \in \mathbb{N}: \sum_{j=0}^{\infty} g_{j}(t) u(t+j)=0 .
\end{gathered}
$$

If in (13) $f_{d}(t) \neq 0$ for all $t$ then the standard recursive solution

$$
w(t+d)=f_{d}(t)^{-1}\left(u(t)-\sum_{i=0}^{d-1} f_{i}(t) w(t+i)\right)
$$

holds. Thm. 2.1 implies that there is a solution $w$ of $f \circ w=u$ too if $f_{d} \in \mathbb{F}^{\mathbb{N}}$ has many zeros provided $u$ satisfies the necessary compatibility conditions $g \circ u=0$ if $g f=0$. Even in this simplest case of Thm. 2.1,(2), the existence of a solution $w$ of $f \circ w=u$ is not obvious. If $f_{d}(0)=0$ the solution $w$ has to satisfy the linear equation

$$
\sum_{i=0}^{d-1} f_{i}(0) w(i)=u(0),\left(f_{d}(0)=0\right)
$$

so the initial values $w(0), \cdots, w(d-1)$ cannot be chosen arbitrarily.

If ${ }_{\mathbf{A}} W^{\prime}$ is any signal module and $U \subseteq \mathbf{A}^{1 \times \ell}$ is a submodule with factor module $M:=\mathbf{A}^{1 \times \ell} / U$ there is the canonical $\mathbb{F}$-isomorphism

$$
\begin{gathered}
\operatorname{Hom}_{\mathbf{A}}\left(M, W^{\prime}\right) \underset{\text { Malgrange 1962 }}{\cong} \mathcal{B}:=U^{\perp}:=\left\{w \in W^{\prime \ell} ; \forall \xi \in U: \xi \circ w=0\right\} \\
\varphi \mapsto w, \varphi(\xi+U)=\xi \circ w, \xi \in \mathbf{A}^{1 \times \ell} .
\end{gathered}
$$

The solution $\mathbb{F}$-module $U^{\perp}$ of all linear equations $\xi \circ w=0, \xi \in U$, is called the $W^{\prime}$-behavior defined by $U$ or $M$, and $U$ resp. $M$ are called the equation module resp. system module of $\mathcal{B}$. If $U$ is finitely generated (f.g.) and $U=\mathbf{A}^{1 \times k} R, R \in \mathbf{A}^{k \times \ell}$, for instance always if $\mathbf{A}$ is a left noetherian ring, then

$$
\mathcal{B}=\left\{w \in W^{\prime \ell} ; R \circ w=0\right\}
$$

These solution modules are ubiquitous in linear systems theory and explain the significance of the functor $\operatorname{Hom}_{\mathbf{A}}\left(-, W^{\prime}\right)$ for this theory. The monoid $\mathbb{F}$-algebra

$$
\mathbb{F}[N]=\oplus_{i \in N} \mathbb{F} q^{i} \subset \mathbf{A}
$$

is a commutative subalgebra of $\mathbf{A}$ and therefore acts on $\mathbb{F}^{N}$ too. For a field [18, Thm. 3.15] resp. for a self-injective ring $\mathbb{F}[15]$ the signal module ${ }_{\mathbb{F}[N]} \mathbb{F}^{N}$ is an injective $\mathbb{F}[N]$-module and hence satisfies the fundamental principle. This follows easily from the canonical $\mathbb{F}[N]$-linear isomorphisms $(M \in \mathbb{F}[N]$ Mod $)$

$$
\begin{gathered}
\operatorname{Hom}_{\mathbb{F}}(\mathbb{F}[N], \mathbb{F}) \underset{\mathbb{F}[N]}{\cong} \mathbb{F}^{N}, \varphi \mapsto\left(\varphi\left(q^{i}\right)\right)_{i \in N} \\
\operatorname{Hom}_{\mathbb{F}[N]}\left(M, \mathbb{F}^{N}\right) \cong \operatorname{Hom}_{\mathbb{F}[N]}\left(M, \operatorname{Hom}_{\mathbb{F}}(\mathbb{F}[N], \mathbb{F})\right) \cong \\
\Phi(x)(i)=\varphi\left(q^{i} x\right), x \in M, i \in N
\end{gathered}
$$


and the self-injectivity of $\mathbb{F}$. In [5] an analogue of injectivity is proven for the noncommutative polynomial algebra $\mathbb{F}(t)[q ; \circ]$ and suitably defined behaviors where $\mathbb{F}$ is a field and $\mathbb{F}(t)$ the field of rational functions in one indeterminate $t$ with the action $(i \circ a)(t):=a(t+i)$. There is no continuous analogue of Thm. 2.1 for differential LTV- systems

$$
\sum_{j=0}^{d} R_{j}(t) \frac{d^{j} w}{d t^{j}}(t)=u(t), t \in \mathbb{R},
$$

and the signal spaces $\mathrm{C}^{\infty}(\mathbb{R})$ or $\mathcal{D}^{\prime}(\mathbb{R})$ of smooth functions or distributions over appropriate operator rings of differential operators with variable coefficients. In the continuous case the signal space has to be enlarged to become injective over the larger ring of differential operators [9], [22], [4].

A module ${ }_{\mathbf{A}} W^{\prime}$ is called a cogenerator if for every $\mathbf{A}$-module $\mathbf{M}$ the canonical $\mathbf{A}$-linear Gelfand map

$$
\begin{aligned}
\rho_{M}: M \rightarrow & \operatorname{Hom}_{\mathbb{F}}\left(\operatorname{Hom}_{\mathbf{A}}\left(M, W^{\prime}\right), W^{\prime}\right), x \mapsto \rho_{M}(x), \rho_{M}(x)(\varphi):=\varphi(x), \\
& \text { is injective or } \bigcap\left\{\operatorname{ker}(\varphi) ; \varphi \in \operatorname{Hom}_{\mathbf{A}}\left(M, W^{\prime}\right)\right\}=0 .
\end{aligned}
$$

The cogenerator property of ${ }_{\mathbf{A}} W^{\prime}$ implies in (17) that $\mathcal{B} \cong \operatorname{Hom}_{\mathbf{A}}\left(M, W^{\prime}\right)$ is nonzero if $M$ is nonzero and that $U=\mathcal{B}^{\perp}:=\left\{\xi \in \mathbf{A}^{1 \times \ell} ; \xi \circ \mathcal{B}=0\right\}$. So $\mathcal{B}$ determines $U$ and $U$ and $\mathcal{B}=U^{\perp}$ contain the same information. If ${ }_{\mathbf{A}} W^{\prime}$ is an injective cogenerator then the functor (9) induces a duality (contravariant equivalence) between the category of f.g. A-left modules $M=\mathbf{A}^{1 \times \ell} / U$ (algebra) and that of $W^{\prime}$-behaviors $U^{\perp}$ (engineering mathematics) [18], and thus enables a strong use of algebraic tools for systems theoretic applications like in the standard LTI-systems theories. But also the injectivity alone like in Thm. 2.1 opens many such opportunities. If $\mathbb{F}$ is a cogenerator ring (20) implies that also $\mathbb{F [ N ]} \mathbb{F}^{N}$ is an injective cogenerator.

Theorem 2.3. (1) For the monoids $N=\mathbb{N}^{r_{1}} \times \mathbb{Z}^{r_{2}}$ from (1) the module a $W$ is not a cogenerator $\left(\mathbf{A}:=\mathbb{F}^{N}[N ; \circ], W:=\mathbb{F}^{N}\right)$. (2) If $N$ is a finitely generated abelian group and $\mathbb{F}$ is a cogenerator ring then ${ }_{\mathbf{A}} W$ is a cogenerator if and only if $N$ is finite.

In Section 4 we treat the case of periodic coefficients. We assume a quasi-Frobenius ring $\mathbb{F}$, the group $N:=\mathbb{Z}^{r}$ and a subgroup $L \subseteq N$ such that $\bar{N}:=N / L$ is finite. This implies $L \cong \mathbb{Z}^{r}$. We identify $\mathbb{F}^{\bar{N}}$ with the subalgebra of $\mathbb{F}^{N}$ of $L$-periodic functions by

$$
\begin{aligned}
\mathbb{F}^{\bar{N}}= & \left\{b \in \mathbb{F}^{N} ; \forall t \in N \forall i \in L: b(t+i)=b(t)\right\} \subset \mathbb{F}^{N}, \\
& a(t):=a(\bar{t}) \text { for } a \in \mathbb{F}^{\bar{N}}, t \in N, \bar{t}:=t+L .
\end{aligned}
$$

The finite group $\bar{N}$ acts on itself by translation and so does $t \in N$ on $\bar{i} \in \bar{N}$ by $t+\bar{i}:=\bar{t}+\bar{i}$. This action implies the action $\circ$ of $N$ on $\mathbb{F}^{\bar{N}}$ given by

$$
(i \circ a)(\bar{t})=a(\bar{t}+\bar{i}), i \in N, a \in \mathbb{F}^{\bar{N}} .
$$

This action gives rise to the skew-group algebra or smash product $[16, \S 1.5 .4]$

$$
\begin{gathered}
\mathbf{A}_{\text {per }}:=\mathbb{F}^{\bar{N}}[N ; \circ]=\oplus_{j \in N} \mathbb{F}^{\bar{N}} q^{j} \text { with }\left(a q^{i}\right)\left(b q^{j}\right)=a(i \circ b) q^{i+j}, i, j \in N, a, b \in \mathbb{F}^{\bar{N}}, \\
\text { obviously } \mathbb{F}^{\bar{N}}[N ; \circ] \subset \mathbb{F}^{N}[N ; \circ] .
\end{gathered}
$$


The bijection $N \cong\left\{q^{i} ; i \in N\right\}, i \mapsto q^{i}$, is a group isomorphism. The ring $\mathbf{A}_{\text {per }}$ has the commutative $\mathbb{F}$-subalgebra

$$
\mathbf{Z}:=\mathbb{F}[L]=\oplus_{j \in L} \mathbb{F} q^{j} \subset \mathbf{A}_{\text {per }} .
$$

If $\beta_{\rho}, \rho=1, \cdots, r$, is a $\mathbb{Z}$-basis of $L \cong \mathbb{Z}^{r}=N$ and

$$
x_{\rho}:=q^{\beta_{\rho}} \text { then } \mathbf{Z}=\mathbb{F}[L]=\mathbb{F}\left[x_{1}, x_{1}^{-1}, \cdots, x_{r}, x_{r}^{-1}\right]
$$

is the Laurent polynomial algebra in the $x_{\rho}$ over $\mathbb{F}$ and thus noetherian since the quasiFrobenius ring $\mathbb{F}$ is noetherian and even artinian [14, Thm. 15.27]. By (20) the signal module $\mathbb{Z}^{L}$ is an injective cogenerator and there is the categorical duality between finitely generated (f.g.) $\mathbb{F}[L]$-modules and $\mathbb{F}[L] \mathbb{F}^{L}$-behaviors that are the usual multidimensional behaviors on the lattice $\mathbb{Z}^{r}$ and governed by linear systems of partial difference equations with constant coefficients. We apply the Morita theory $[14, \S 18]$ to show that there is a canonical categorical equivalence ${ }_{\mathbf{A}_{\mathrm{per}}} \operatorname{Mod} \cong{ }_{\mathbf{z}} \mathbf{M o d}$. This implies that not only $\mathbf{Z}^{L}$, but also $\mathbf{A}_{\text {per }} \mathbb{F}^{N}$ is an injective cogenerator. Moreover every $\mathbf{A}_{\text {per }} \mathbb{F}^{N}$-behavior $\mathcal{B}$ is canonically isomorphic to its stacked form $\widehat{\mathcal{B}}$ that is a $\mathbf{z} \mathbb{F}^{L}$ behavior whose kernel representation we describe constructively. The main results are Thms. 4.4, 4.6 and 4.7. In Cor. 4.13 we also consider the case of periodic trajectories (cf. [23]).

\section{The proofs of Thms. 2.1 and 2.3}

\section{1 $\mathbb{F}^{N}$ is injective over $\mathbf{A}=\mathbb{F}^{N}[N ; \circ]$}

The assumptions of Section 2 concerning $N$ and $\mathbb{F}$ are in force. We need several preparations concerning infinite-dimensional free modules that are exposed, for instance, in $[4, \S 3.5 .2 .2]$. If $V$ is an $\mathbb{F}$-module and $\Lambda$ any index set then

$$
V^{\Lambda}:=\left\{w=(w(\lambda))_{\lambda \in \Lambda}: w: \Lambda \rightarrow V\right\}
$$

is the $\mathbb{F}$-module of all functions $\Lambda \rightarrow V$. The support of $w \in V^{\Lambda}$ is the set

$$
\operatorname{supp}(w):=\{\lambda \in \Lambda ; w(\lambda) \neq 0\} . \text { Then } V^{(\Lambda)}:=\left\{w \in V^{\Lambda} ; \operatorname{supp}(w) \text { finite }\right\} \subseteq V^{\Lambda}
$$

is a submodule. The following equations (30)-(33) hold for any ring $\mathbf{A}$. The left $\mathbf{A}$ module $\mathbf{A}^{(\Lambda)}$ is free and has the $\mathbf{A}$-basis

$$
\delta_{\lambda}, \lambda \in \Lambda, \delta_{\lambda}(\mu)=\delta_{\lambda, \mu}:=\left\{\begin{array}{ll}
1 & \text { if } \lambda=\mu \\
0 & \text { if } \lambda \neq \mu
\end{array}, \mathbf{A}^{(\Lambda)}=\oplus_{\lambda \in \Lambda} \mathbf{A} \delta_{\lambda} .\right.
$$

If $\Gamma$ is another set with its associated free module $\mathbf{A}^{(\Gamma)}$ there is the canonical isomorphism between linear maps and matrices given by

$$
\begin{gathered}
\operatorname{Hom}_{\mathbf{A}}\left(\mathbf{A}^{(\Gamma)}, \mathbf{A}^{(\Lambda)}\right) \cong \\
\left\{R=(R(\gamma, \lambda))_{\gamma, \lambda} \in \mathbf{A}^{\Gamma \times \Lambda} ; \forall \gamma \in \Gamma: \operatorname{supp}(R(\gamma,-)) \text { finite }\right\}, \Phi=: \circ R \leftrightarrow R \\
\text { where } \operatorname{supp}(R(\gamma,-)):=\{\lambda \in \Lambda ; R(\gamma, \lambda) \neq 0\}, \\
\forall \gamma \in \Gamma: \Phi\left(\delta_{\gamma}\right)=\sum_{\lambda \in \Lambda} R(\gamma, \lambda) \delta_{\lambda}, \forall \eta \in \mathbf{A}^{(\Gamma)}:(\eta R)(\lambda)=\sum_{\gamma \in \Gamma} \eta(\gamma) R(\gamma, \lambda) .
\end{gathered}
$$


The $\Gamma \times \Lambda$-matrices with finite $\operatorname{supp}(R(\gamma,-))$ for all $\gamma \in \Gamma$ are called row-finite. In the sequel we choose one such row-finite matrix $R \in \mathbf{A}^{\Gamma \times \Lambda}$.

For every $M \in{ }_{\mathbf{A}}$ Mod with action $\circ$ there is the canonical isomorphism

$$
\begin{gathered}
\operatorname{Hom}_{\mathbf{A}}\left(A^{(\Gamma)}, M\right) \cong M^{\Gamma}, \Phi \mapsto\left(\Phi\left(\delta_{\gamma}\right)\right)_{\gamma \in \Gamma} \text { and we identify } \\
\operatorname{Hom}_{\mathbf{A}}\left(A^{(\Gamma)}, M\right)=M^{\Gamma}, \Phi=\left(\Phi\left(\delta_{\gamma}\right)\right)_{\gamma \in \Gamma} .
\end{gathered}
$$

With this identification the row-finite matrix $R$ from (31) gives rise to the adjoint map

$$
R \circ:=\operatorname{Hom}(\circ R, M): M^{\Lambda} \rightarrow M^{\Gamma},(R \circ w)(\gamma)=\sum_{\lambda \in \Lambda} R(\gamma, \lambda) \circ w(\lambda), \gamma \in \Gamma .
$$

This applies especially to the A-module $W=\mathbb{F}^{N}$. We identify

$$
W^{\Gamma}=\left(\mathbb{F}^{N}\right)^{\Gamma}=\left(\mathbb{F}^{\Gamma}\right)^{N}=\mathbb{F}^{\Gamma \times N}, w(\gamma)(t)=w(t)(\gamma)=w(\gamma, t), \gamma \in \Gamma, t \in N .
$$

The elements $R(\gamma, \lambda)$ have the form

$$
R(\gamma, \lambda)=\sum_{j \in N} R(\gamma, \lambda)_{j} q^{j}, \forall j, t \in N: R(\gamma, \lambda)_{j} \in \mathbb{F}^{N}, R(\gamma, \lambda)_{j}(t) \in \mathbb{F} .
$$

For

$$
\begin{gathered}
w=(w(\lambda))_{\lambda \in \Lambda}=(w(\lambda, j))_{(\lambda, j) \in \Lambda \times N} \in W^{\Lambda}, w(\lambda)(j)=w(\lambda, j), \text { and } \\
u:=R \circ w=(u(\gamma))_{\gamma \in \Gamma}=(u(\gamma, i))_{(\gamma, i) \in \Gamma \times N} \in W^{\Gamma}
\end{gathered}
$$

we get

$$
\begin{aligned}
u(\gamma) & =\sum_{\lambda \in \Lambda} R(\gamma, \lambda) \circ w(\lambda), u(\gamma, i)=\sum_{(\lambda, j) \in \Lambda \times N} R(\gamma, \lambda)_{j}(i) w(\lambda)(i+j) \\
& =\sum_{(\lambda)=i+j} R(\gamma, \lambda)_{k-i}(i) w(\lambda)(k) \\
& =\sum_{(\lambda, k) \in \Lambda \times N \times N} H((\gamma, i),(\lambda, k)) w(\lambda, k)=(H \cdot w)(\gamma, i)
\end{aligned}
$$

where

$$
\begin{gathered}
H((\gamma, i),(\lambda, k)):=\left\{\begin{array}{ll}
R(\gamma, \lambda)_{k-i}(i) & \text { if } k \in i+N \\
0 & \text { if } k \notin i+N
\end{array},\right. \\
H=\left(H((\gamma, i),(\lambda, k))_{((\gamma, i),(\lambda, k)) \in(\Gamma \times N) \times(\Lambda \times N)} \in \mathbb{F}^{(\Gamma \times N) \times(\Lambda \times N)} .\right.
\end{gathered}
$$

We conclude

$$
\forall w \in W^{\Lambda}=\mathbb{F}^{\Lambda \times N}: R \circ w=H \cdot w \in W^{\Gamma}=\mathbb{F}^{\Gamma \times N} .
$$

Here we use the notation $H \cdot w$ for multiplication of infinite matrices with entries in $\mathbb{F}$. Notice that the matrix $H$ is row-finite since for fixed $(\gamma, i) \in \Gamma \times N$ almost all, up to finitely many, $R(\gamma, \lambda)$ and almost all $R(\gamma, \lambda)_{j}$ are zero. The significance of equation (39) lies in the fact that it replaces the operator product $R \circ w$ by the matrix 
multiplication $H \cdot w$ of matrices with coefficients in $\mathbb{F}$. The matrix $H$ gives rise to the exact sequence of $\mathbb{F}$-modules and $\mathbb{F}$-linear maps

$$
\begin{gathered}
\operatorname{ker}(\cdot H) \stackrel{\text { inj }}{\longrightarrow} \mathbb{F}^{(\Gamma \times N)} \stackrel{\cdot H}{\longrightarrow} \mathbb{F}^{(\Lambda \times N)} \text { with } \operatorname{inj}(\eta)=\eta, \\
(\eta \cdot H)(\lambda, j)=\sum_{(\gamma, i) \in \Gamma \times N} \eta(\gamma, i) H((\gamma, i),(\lambda, j)), \eta \in \mathbb{F}^{(\Gamma \times N)}, \eta: \Gamma \times N \rightarrow \mathbb{F} .
\end{gathered}
$$

Recall that we identify $\operatorname{Hom}_{\mathbb{F}}\left(\mathbb{F}^{(I)}, \mathbb{F}\right)=\mathbb{F}^{I}$ (cf. (32)). Since $\mathbb{F} \mathbb{F}$ is assumed injective the duality functor

$$
\operatorname{Hom}_{\mathbb{F}}(-, \mathbb{F}): \mathbb{F} \mathbf{M o d} \rightarrow{ }_{\mathbb{F}} \operatorname{Mod}, U \mapsto U^{*}:=\operatorname{Hom}_{\mathbb{F}}(U, \mathbb{F}),
$$

is exact. Applied to the exact sequence (40) of $\mathbb{F}$-modules it furnishes the exact sequence of $\mathbb{F}$-modules

$$
\begin{gathered}
W^{\Lambda}=\mathbb{F}^{\Lambda \times N} \stackrel{H \cdot}{\longrightarrow} W^{\Gamma}=\mathbb{F}^{\Gamma \times N} \stackrel{\text { res }}{\longrightarrow} \operatorname{ker}(\cdot H)^{*} \text { with } \\
\text { res }:=\operatorname{Hom}(\operatorname{inj}, \mathbb{F}): \mathbb{F}^{\Gamma \times N} \rightarrow \operatorname{ker}(\cdot H)^{*}, u \mapsto u \mid \operatorname{ker}(\cdot H),
\end{gathered}
$$

where $u=(u(\gamma, i))_{(\gamma, i) \in \Gamma \times N}$ and $u \mid \operatorname{ker}(\cdot H)$ has the form

$$
\begin{gathered}
u \mid \operatorname{ker}(\cdot H): \eta \mapsto \sum_{(\gamma, i) \in \Gamma \times N} \eta(\gamma, i) u(\gamma, i) \text { for } \eta \in \operatorname{ker}(\cdot H) \subseteq \mathbb{F}^{(\Gamma \times N)} \text { with } \\
\eta \cdot H=0 \text { or } \forall(\lambda, j) \in \Lambda \times N: \sum_{(\gamma, i) \in \Gamma \times N} \eta(\gamma, i) H((\gamma, i),(\lambda, j))=0 .
\end{gathered}
$$

Proof. (of Thm. 2.1) (1) (i) Let $X \subset \mathbf{A}^{(\Lambda)}$ be any submodule. Choose a row-finite matrix $R \in \mathbf{A}^{\Gamma \times \Lambda}$ such that $X=\sum_{\gamma \in \Gamma} \mathbf{A} R(\gamma,-) \subseteq \mathbf{A}^{(\Lambda)}$. Let $\varphi \in \operatorname{Hom}_{\mathbf{A}}(X, W)$ be any $\mathbf{A}$-linear map. We show in (ii) below that $\varphi$ can be extended to an $\mathbf{A}$-linear map $\psi: \mathbf{A}^{(\Lambda)} \rightarrow W$ with $\psi \mid X=\varphi$. For $\Lambda=\{1\}$ and $\mathbf{A}^{(\Lambda)}=\mathbf{A}$ the submodule $X$ is a left ideal of $\mathbf{A}$ with generators $R(\gamma, 1), \gamma \in \Gamma$. According to Baer's criterion for injectivity [14, Thm. 3.7] the extendability of each A-linear map $\varphi: X \rightarrow W$ to an A-linear map $\psi: \mathbf{A} \rightarrow W$ implies the injectivity of $\mathbf{A}_{\mathbf{A}} W$.

(ii) We apply the exact sequence (42) to the chosen $R$. Define $u \in W^{\Gamma}$ by $u(\gamma):=$ $\varphi(R(\gamma,-))$. In (iii) below we show that $u \mid \operatorname{ker}(\cdot H)=0$. From this, (42) and (39) we conclude that there is $w \in W^{\Lambda}$ such that

$$
\begin{gathered}
u=H \cdot w=R \circ w \text { or } \forall \gamma \in \Gamma: u(\gamma)=\sum_{\lambda \in \Lambda} R(\gamma, \lambda) \circ w(\lambda) \text {. Define } \\
\psi: \mathbf{A}^{(\Lambda)} \rightarrow W \text { by } \psi\left(\delta_{\lambda}\right)=w(\lambda) \Longrightarrow \\
\psi(R(\gamma,-))=\psi\left(\sum_{\lambda} R(\gamma, \lambda) \delta_{\lambda}\right)=\sum_{\lambda} R(\gamma, \lambda) \circ \psi\left(\delta_{\lambda}\right)= \\
\sum_{\lambda} R(\gamma, \lambda) \circ w(\lambda)=u(\gamma)=\varphi(R(\gamma,-)) \Longrightarrow \psi \mid X=\varphi
\end{gathered}
$$

since the $R(\gamma,-)$ generate $X$.

(iii) It remains to show that $u \mid \operatorname{ker}(\cdot H)=0$. Let $\xi \in \operatorname{ker}(\cdot H) \subseteq \mathbb{F}^{(\Gamma \times N)}$. Consider 
$\delta_{0} \in \mathbb{F}^{N}$ with $\delta_{0}(t)=\delta_{0, t}$. Define

$$
\begin{aligned}
\eta \in \mathbf{A}^{(\Gamma)} \text { by } \eta(\gamma)=\sum_{i \in N} \xi(\gamma, i) \delta_{0} q^{i} \in \mathbf{A} \Longrightarrow(\eta R)(\lambda)=\sum_{\gamma \in \Gamma} \eta(\gamma) R(\gamma, \lambda) \\
=\sum_{\gamma \in \Gamma, i \in N, j \in N} \xi(\gamma, i) \delta_{0} q^{i} R(\gamma, \lambda)_{j} q^{j}=\sum_{\gamma \in \Gamma, i \in N, j \in N} \xi(\gamma, i) \delta_{0}\left(i \circ R(\gamma, \lambda)_{j}\right) q^{i+j} \\
=\sum_{k \in N}\left(\sum_{(\gamma, i) \in \Gamma \times N \text { with } k \in i+N} \xi(\gamma, i) \delta_{0}\left(i \circ R(\gamma, \lambda)_{k-i}\right)\right) q^{k} .
\end{aligned}
$$

We show $\eta R=0$ by proving that for all $\lambda \in \Lambda$ and all $k \in N$ the coefficient of $q^{k}$ in $(\eta R)(\lambda) \in \mathbf{A}=\mathbb{F}^{N}[N ; \circ]$ is zero. But for all $k, t \in N$

$$
\begin{aligned}
& \left(\sum_{(\gamma, i) \in \Gamma \times N \text { with } k \in i+N} \xi(\gamma, i) \delta_{0}\left(i \circ R(\gamma, \lambda)_{k-i}\right)\right)(t) \\
& =\sum_{(\gamma, i) \in \Gamma \times N \text { with } k \in i+N} \xi(\gamma, i) \delta_{0}(t) R(\gamma, \lambda)_{k-i}(t+i) \\
& = \begin{cases}0 & \text { if } t \neq 0 \\
\sum_{(\gamma, i) \in \Gamma \times N, k \in i+N} \xi(\gamma, i) R(\gamma, \lambda)_{k-i}(i) & \text { if } t=0\end{cases} \\
& = \begin{cases}0 & \text { if } t \neq 0 \\
\sum_{(\gamma, i) \in \Gamma \times N} \xi(\gamma, i) H((\gamma, i),(\lambda, k))=(\xi \cdot H)(\lambda, k) \underset{\xi \in \operatorname{ker}(\cdot H)}{=} 0 & \text { if } t=0\end{cases} \\
& \Longrightarrow \eta R=0 .
\end{aligned}
$$

From $\eta R=0$ we now infer

$$
\begin{aligned}
\eta \circ u & =\sum_{\gamma \in \Gamma} \eta(\gamma) \circ u(\gamma)=\sum_{\gamma \in \Gamma} \eta(\gamma) \circ \varphi(R(\gamma,-)) \\
& \left.=\varphi\left(\sum_{\gamma \in \Gamma} \eta(\gamma) R(\gamma,-)\right)\right)=\varphi(\eta R) \underset{\eta R=0}{=} \varphi(0)=0 \\
\Longrightarrow 0 & =\left(\sum_{\gamma \in \Gamma} \eta(\gamma) \circ u(\gamma)\right)(0)=\sum_{(\gamma, i) \in \Gamma \times N} \xi(\gamma, i) \delta_{0}(0) u(\gamma)(0+i) \\
& =\sum_{(\gamma, i) \in \Gamma \times N} \xi(\gamma, i) u(\gamma, i) \underset{\forall \xi \in \operatorname{ker}(\cdot H)}{\Longrightarrow} u \mid \operatorname{ker}(\cdot H)=0 .
\end{aligned}
$$

(2) Part (2) of Thm. 2.1 is proven in the more general Cor. 3.1.

Corollary 3.1. (cf. [4, Cor. and Def. 592, Remark 593]) If $R \in \mathbf{A}^{\Gamma \times \Lambda}$ is any row-finite matrix and $u \in W^{\Gamma}$ is any right side the linear system

$$
R \circ w=u, w \in W^{\Lambda}, \text { or } \forall \gamma \in \Gamma: \sum_{\lambda \in \Lambda} R(\gamma, \lambda) \circ w(\lambda)=u(\gamma)
$$


has a solution $w \in W^{\Lambda}$ if and only if $u$ satisfies the canonical compatibility conditions

$$
\begin{gathered}
\forall \eta \in \mathbf{A}^{(\Gamma)}: \\
\left(\eta R=0 \text {, i.e., } \forall \lambda \in \Lambda: \sum_{\gamma \in \Gamma} \eta(\gamma) R(\gamma, \lambda)=0 \Longrightarrow \eta \circ u=\sum_{\gamma \in \Gamma} \eta(\gamma) \circ u(\gamma)=0\right) .
\end{gathered}
$$

Proof. Recall that we identify $\operatorname{Hom}_{\mathbf{A}}\left(\mathbf{A}^{(\Lambda)}, W\right)=W^{\Lambda}$ and $R \circ=\operatorname{Hom}(\circ R, W)$. Let

$$
\begin{aligned}
& U:=\operatorname{ker}\left(\circ R: \mathbf{A}^{(\Gamma)} \rightarrow \mathbf{A}^{(\Lambda)}\right)=\left\{\eta \in \mathbf{A}^{(\Gamma)} ; \eta R=0\right\}
\end{aligned}
$$

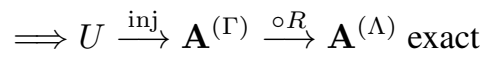

$$
\begin{aligned}
& W \text { injective }, \operatorname{Hom}_{\mathbf{A}}(-, W) \text { exact } W^{\Lambda} \stackrel{R \circ}{\longrightarrow} W^{\Gamma} \stackrel{\operatorname{Hom}(\operatorname{inj}, W)}{\longrightarrow} \operatorname{Hom}_{\mathbf{A}}(U, W) \text { exact }
\end{aligned}
$$

where $\operatorname{inj}(\eta)=\eta$ and $\operatorname{Hom}(\operatorname{inj}, W): u \mapsto(\eta \mapsto \eta \circ u)$. As asserted we conclude

$R \circ W^{\Lambda}=\operatorname{im}(R \circ)=\operatorname{ker}(\operatorname{Hom}(\operatorname{inj}, W))=U^{\perp}=\left\{u \in W^{\Gamma} ; \forall \eta \in U ; \eta \circ u=0\right\}$.

\section{2 $\mathbb{F}^{N}$ is not a cogenerator}

We use a commutative self-injective ring $\mathbb{F}$ as before and a monoid $N=\mathbb{N}^{r_{1}} \times \mathbb{Z}^{r_{2}}$ from (1). We prove Theorem 2.3 by a counter-example with a suitably chosen

$$
f \in \mathbf{A} \text { with } \mathbf{A} / \mathbf{A} f \neq 0, \text { but } \operatorname{Hom}_{\mathbf{A}}(\mathbf{A} / \mathbf{A} f, W) \cong\{w \in W ; f \circ w=0\}=0 .
$$

The part (2) of Thm. 2.3 will be shown in Cor. 4.14.

(1) We first assume $N=\mathbb{N}$ and $\mathbf{A}=\oplus_{i \in \mathbb{N}} \mathbb{F} q^{i}$. In this case we interpret $q:=q^{1}$ as an indeterminate and $q^{i}=\left(q^{1}\right)^{i}$ as its $i$-th power.

(i) Below we choose a suitable nonzero $f_{1} \in \mathbb{F}^{\mathbb{N}}$ and define the skew-polynomial $f:=f_{1} q-1 \in \mathbf{A}$ of $q$-degree 1 such that the properties of (52) are satisfied and thus refute the cogenerator property of ${ }_{\mathbf{A}} W$. It is obvious that $\mathbf{A}=\mathbf{A} f$ if and only if there is a $g=\sum_{i=0}^{d} g_{i} q^{i}$ with $g f=1$. But

$$
\begin{aligned}
g f & =\sum_{i=0}^{d} g_{i} q^{i}\left(f_{1} q-1\right)=\sum_{i=0}^{d} g_{i}\left(i \circ f_{1}\right) q^{i+1}-\sum_{i=0}^{d} g_{i} q^{i} \\
& =g_{d}\left(d \circ f_{1}\right) q^{d+1}+\sum_{i=1}^{d}\left(g_{i-1}\left((i-1) \circ f_{1}\right)-g_{i}\right) q^{i}-g_{0} .
\end{aligned}
$$

We conclude that

$$
\begin{aligned}
& g f=1 \Longleftrightarrow g_{0}=-1, \forall i=1, \cdots, d: g_{i}=g_{i-1}\left((i-1) \circ f_{1}\right), g_{d}\left(d \circ f_{1}\right)=0 \\
& \quad \Longleftrightarrow g_{0}=-1, \forall i=1, \cdots, d: g_{i}=-\left((i-1) \circ f_{1}\right) * \cdots *\left(1 \circ f_{1}\right) * f_{1} \text { and } \\
& \left(d \circ f_{1}\right) * \cdots *\left(1 \circ f_{1}\right) * f_{1}=0 .
\end{aligned}
$$


Hence

$$
\begin{aligned}
\mathbf{A}=\mathbf{A} f & \Longleftrightarrow \exists d \geq 1 \text { with }\left(d \circ f_{1}\right) * \cdots *\left(1 \circ f_{1}\right) * f_{1}=0 \\
& \Longleftrightarrow \exists d \geq 1 \forall t \in \mathbb{N}: f_{1}(t+d) * f_{1}(t+d-1) * \cdots * f_{1}(t)=0 .
\end{aligned}
$$

(ii) We define $f_{1} \in \mathbb{F}^{\mathbb{N}}$ and $f$ by

$$
f_{1}(t):=\left\{\begin{array}{ll}
1 & \text { if } t \neq 2^{n}, n \in \mathbb{N} \\
0 & \text { if } t=2^{n}
\end{array}, f:=f_{1} q-1 .\right.
$$

For arbitrary $d \geq 1$ choose $n$ with $1 \leq d<2^{n}-1$ and $t:=2^{n}+1$. Then

$$
\begin{aligned}
& 2^{n}<t=2^{n}+1 \leq t+d<2^{n}+1+2^{n}-1=2^{n+1} \\
& \Longrightarrow f_{1}(t+d)=\cdots=f_{1}(t)=1 \\
& \Longrightarrow f_{1}(t+d) f_{1}(t+d-1) * \cdots * f_{1}(t+1) * f_{1}(t)=1 .
\end{aligned}
$$

Equations (55) and (57) imply $\mathbf{A} f \subsetneq \mathbf{A}$ and $M:=\mathbf{A} / \mathbf{A} f \neq 0$.

(iii) For $f=f_{1} q-1$ from (56) we show that the associated behavior

$$
\mathcal{B}:=\left\{w \in \mathbb{F}^{\mathbb{N}} ; f \circ w=0\right\}=\left\{w \in \mathbb{F}^{\mathbb{N}} ; \forall t \in \mathbb{N}: f_{1}(t) w(t+1)=w(t)\right\}
$$

is zero. By decreasing induction the equation $w(t-1)=f_{1}(t-1) w(t), t \geq 1$, shows that $w\left(t_{0}\right)=0$ for some $t_{0}$ implies $w(t)=0$ for all $t \leq t_{0}$. But for all $n$ and $w \in \mathcal{B}$ we have

$$
\begin{gathered}
0=f_{1}\left(2^{n}\right) w\left(2^{n}+1\right)-w\left(2^{n}\right)=0 w\left(2^{n}+1\right)-w\left(2^{n}\right) \Longrightarrow w\left(2^{n}\right)=0 \Longrightarrow \\
\forall n \forall t \leq 2^{n}: w(t)=0 \Longrightarrow w=0 \Longrightarrow \mathcal{B}=0 .
\end{gathered}
$$

(2) (i) In the next step we assume $N=\mathbb{Z}$ and define $f_{1}^{\mathbb{Z}} \in \mathbb{F}^{\mathbb{Z}}$ with $f_{1} \in \mathbb{F}^{\mathbb{N}}$ from (56) by

$$
\begin{gathered}
f_{1}^{\mathbb{Z}}(t):=\left\{\begin{array}{ll}
f_{1}(t) & \text { if } t \geq 0 \\
0 & \text { if } t<0
\end{array}, f^{\mathbb{Z}}:=f_{1}^{\mathbb{Z}} q-1 \in \mathbf{A}^{\mathbb{Z}}:=\mathbb{F}^{\mathbb{Z}}\left[q, q^{-1} ; \circ\right],\right. \\
\mathcal{B}^{\mathbb{Z}}:=\left\{w \in \mathbb{F}^{\mathbb{Z}} ; f^{\mathbb{Z}} \circ w=0\right\}=\left\{w \in \mathbb{F}^{\mathbb{Z}} ; \forall t \in \mathbb{Z}: f_{1}^{\mathbb{Z}}(t) w(t+1)=w(t)\right\} .
\end{gathered}
$$

We infer that $w\left(t_{0}\right)=0$ for some $t_{0} \in \mathbb{Z}$ implies $w(t)=0$ for all $t \leq t_{0}$. But $f_{1}^{\mathbb{Z}}\left(2^{n}\right)=f_{1}\left(2^{n}\right)=0$ and $0=f_{1}^{\mathbb{Z}}\left(2^{n}\right) w\left(2^{n}+1\right)-w\left(2^{n}\right)=w\left(2^{n}\right)$ imply $w\left(2^{n}\right)=0$ for all $n \in \mathbb{N}$ and therefore $w=0$ and $\mathcal{B}^{\mathbb{Z}}=0$.

(ii) We show $\mathbf{A}^{\mathbb{Z}} f^{\mathbb{Z}} \subsetneq \mathbf{A}^{\mathbb{Z}}$ : Assume on the contrary that $g^{\mathbb{Z}}=\sum_{j \in \mathbb{Z}} g_{j}^{\mathbb{Z}} q^{j} \in \mathbf{A}^{\mathbb{Z}}$ with $g^{\mathbb{Z}} f^{\mathbb{Z}}=1$. A slightly simpler computation than (1)(i) above implies

$$
\forall j \in \mathbb{Z}: g_{j-1}^{\mathbb{Z}}\left((j-1) \circ f_{1}^{\mathbb{Z}}\right)=g_{j}^{\mathbb{Z}}+\delta_{j, 0} .
$$

Since almost all $g_{j}^{\mathbb{Z}}$ are zero there is some $n<0$ such that $g_{n}^{\mathbb{Z}}=0$. Equation (61) implies by increasing induction from $n$ to 0 that

$$
\begin{gathered}
\forall j<0: g_{j}^{\mathbb{Z}}=0, g_{0}^{\mathbb{Z}}=-1 \text {. Define } \forall j \geq 0: g_{j}:=g_{j}^{\mathbb{Z}} \mid \mathbb{N} \in \mathbb{F}^{\mathbb{N}} \overrightarrow{\overline{(61)}} \\
g_{0}=-1, \forall j \geq 1: g_{j-1}\left((j-1) \circ f_{1}\right)=g_{j} \underset{(1)(i)}{\Longrightarrow}\left(\sum_{j \in \mathbb{N}} g_{j} q^{j}\right)\left(f_{1} q-1\right)=1 .
\end{gathered}
$$


This contradicts (1)(ii) and hence $\mathbf{A}^{\mathbb{Z}} f^{\mathbb{Z}} \subsetneq \mathbf{A}^{\mathbb{Z}}$. We conclude $\mathbf{A}^{\mathbb{Z}} / \mathbf{A}^{\mathbb{Z}} f^{\mathbb{Z}} \neq 0$, but $\mathcal{B}^{\mathbb{Z}} \cong \operatorname{Hom}_{\mathbf{A}^{\mathbb{Z}}}\left(\mathbf{A}^{\mathbb{Z}}, \mathbb{F}^{\mathbb{Z}}\right)=0$ and therefore that $\mathbb{F}^{\mathbb{Z}}$ is not an $\mathbf{A}^{\mathbb{Z}}$-cogenerator.

(3) (i) Let

$$
\begin{gathered}
N=N_{1} \times N_{2} \ni t=\left(t_{1}, t_{2}\right), i=\left(i_{1}, i_{2}\right), \\
\mathbf{A}_{k}:=\mathbb{F}^{N_{k}}\left[N_{k} ; \circ\right]=\oplus_{i_{k} \in N_{k}} \mathbb{F}^{\mathbb{N}_{k}} q_{k}^{i_{k}}, k=1,2, \\
\mathbf{A}=\mathbb{F}^{N_{1} \times N_{2}}\left[N_{1} \times N_{2} ; \circ\right]=\oplus_{i=\left(i_{1}, i_{2}\right) \in N} \mathbb{F}^{N} q^{i}, q^{\left(i_{1}, i_{2}\right)}=q_{1}^{i_{1}} q_{2}^{i_{2}} \\
W=\mathbb{F}^{N_{1} \times N_{2}}=\left(\mathbb{F}^{N_{1}}\right)^{N_{2}} .
\end{gathered}
$$

We assume that there is an $f=\sum_{j_{1} \in N_{1}} f_{j_{1}} q_{1}^{j_{1}} \in \mathbf{A}_{1}$ such that $\mathbf{A}_{1} f \subsetneq \mathbf{A}_{1}$, but

$$
\begin{gathered}
0=\mathcal{B}_{1}:=\left\{w_{1} \in \mathbb{F}^{N_{1}} ; f \circ w_{1}=0\right\}= \\
\left\{w_{1} \in \mathbb{F}^{N_{1}} ; \forall t_{1} \in N_{1}: \sum_{j_{1} \in N_{1}} f_{j_{1}}\left(t_{1}\right) w\left(t_{1}+j_{1}\right)=0\right\} \cong \\
\operatorname{Hom}_{\mathbf{A}_{1}}\left(\mathbf{A}_{1} / \mathbf{A}_{1} f, \mathbb{F}^{N_{1}}\right) .
\end{gathered}
$$

This assumption implies that $\mathbf{A}_{1} \mathbb{F}^{N_{1}}$ is not a cogenerator. In (1) and (2) above we have constructed such an $f$ for the case that $N_{1}=\mathbb{N}$ or $N_{1}=\mathbb{Z}$. We are going to derive the same for $\mathbb{A}^{N}$. Define

$$
F=\sum_{j_{1} \in N_{1}} F_{j_{1}} q_{1}^{j_{1}} \in \mathbf{A}, \forall j_{1} \in N_{1} \forall t=\left(t_{1}, t_{2}\right) \in N: F_{j_{1}}\left(t_{1}, t_{2}\right):=f_{j_{1}}\left(t_{1}\right) .
$$

We consider the behavior

$$
\begin{gathered}
\mathcal{B}:=\left\{w \in \mathbb{F}^{N} ; F \circ w=0\right\}= \\
\left\{w \in \mathbb{F}^{N} ; \forall\left(t_{1}, t_{2}\right) \in N_{1} \times N_{2}: \sum_{j_{1} \in N_{1}} F_{j_{1}}\left(t_{1}, t_{2}\right) w\left(t_{1}+j_{1}, t_{2}\right)=0\right\}= \\
\left\{w \in \mathbb{F}^{N} ; \forall\left(t_{1}, t_{2}\right) \in N_{1} \times N_{2}: \sum_{j_{1} \in N_{1}} f_{j_{1}}\left(t_{1}\right) w\left(t_{1}+j_{1}, t_{2}\right)=0\right\} \Longrightarrow \\
\forall w \in \mathcal{B} \forall t_{2} \in N_{2}:\left(w\left(t_{1}, t_{2}\right)\right)_{t_{1} \in N_{1}} \in \mathcal{B}_{1} .
\end{gathered}
$$


Since $\mathcal{B}_{1}=0$ this implies that also $\mathcal{B}=0$.

(ii) We show next that $\mathbf{A} F \subsetneq \mathbf{A}$. Assume

$$
\begin{aligned}
& G=\sum_{\left(i_{1}, i_{2}\right) \in N} G_{i_{1}, i_{2}} q_{1}^{i_{1}} q_{2}^{i_{2}} \in \mathbf{A} \text { with } \\
& 1=G F=\sum_{i_{1}, i_{2}, j_{1}} G_{i_{1}, i_{2}}\left(\left(i_{1}, i_{2}\right) \circ F_{j_{1}}\right) q^{\left(i_{1}+j_{1}, i_{2}\right)} \\
& \underset{i_{1}+\overline{j_{1}}=k_{1}}{=} \sum_{\left(k_{1}, i_{2}\right) \in N}\left(\sum_{i_{1}+j_{1}=k_{1}} G_{i_{1}, i_{2}}\left(\left(i_{1}, i_{2}\right) \circ F_{j_{1}}\right)\right) q^{\left(k_{1}, i_{2}\right)} \\
& \Longrightarrow \forall\left(k_{1}, i_{2}\right) \in N: \sum_{i_{1}+j_{1}=k_{1}} G_{i_{1}, i_{2}}\left(\left(i_{1}, i_{2}\right) \circ F_{j_{1}}\right)=\delta_{\left(k_{1}, i_{2}\right),(0,0)} \\
& \Longrightarrow \forall\left(k_{1}, i_{2}\right),\left(t_{1}, t_{2}\right) \in N \text { : } \\
& \sum_{i_{1}+j_{1}=k_{1}} G_{i_{1}, i_{2}}\left(t_{1}, t_{2}\right) f_{j_{1}}\left(t_{1}+i_{1}\right)=\delta_{\left(k_{1}, i_{2}\right),(0,0)} \\
& \underset{i_{2}=t_{2}:=0}{\Longrightarrow} \forall k_{1}, t_{1} \in N_{1}: \sum_{i_{1}+j_{1}=k_{1}} G_{i_{1}, 0}\left(t_{1}, 0\right) f_{j_{1}}\left(t_{1}+i_{1}\right)=\delta_{k_{1}, 0} \\
& \Longrightarrow \text { with } g_{i_{1}}\left(t_{1}\right):=G_{\left(i_{1}, 0\right)}\left(t_{1}, 0\right), g_{i_{1}} \in \mathbb{F}^{N_{1}} \text { : } \\
& \left(\sum_{i_{1} \in N_{1}} g_{i_{1}} q_{1}^{i_{1}}\right)\left(\sum_{j_{1} \in N_{1}} f_{j_{1}} q_{1}^{j_{1}}\right)=\left(\sum_{i_{1} \in N_{1}} g_{i_{1}} q_{1}^{i_{1}}\right) f=1 \Longrightarrow \mathbf{A}_{1} f=\mathbf{A}_{1} \text {. }
\end{aligned}
$$

This contradicts the assumption $\mathbf{A}_{1} f \subsetneq \mathbf{A}_{1}$ and hence

$$
\mathbf{A} F \subsetneq \mathbf{A} \text {, but } \operatorname{Hom}_{\mathbf{A}}\left(\mathbf{A} / \mathbf{A} F, \mathbb{F}^{N}\right) \cong \mathcal{B}=0 .
$$

This implies that also $\mathbb{A}^{N}$ is not a cogenerator. If $N=\mathbb{N}^{r_{1}} \times \mathbb{Z}^{r_{2}}$ from (1) and $r_{1}+r_{2}>0$ then $N$ admits a representation $N=$ $N_{1} \times N_{2}$ where $N_{1}=\mathbb{N}$ or $N_{1}=\mathbb{Z}$. The application of (1), (2) and (3) then implies that $\mathbb{F}^{N}$ is not an $\mathbb{F}^{N}[N ;$ o $]$-cogenerator.

\section{Partial difference equations with periodic coefficients over quasi-Frobenius rings}

\subsection{Morita equivalence}

The assumptions and notations are those from (23)-(27). For notational simplicity we write again $\mathbf{A}:=\mathbf{A}_{\mathrm{per}}$. The algebra $\mathbf{A}=\mathbb{F}^{N}$ from Section 3 is not used in the remainder of the paper.

The elements of $N=\mathbb{Z}^{r}$ are $t, i, j, k, \ell, m$ etc., the elements of $\bar{N}$ are $\bar{t}:=t+$ $L, \bar{i}, \bar{j}, \bar{k}, \bar{\ell}, \bar{m}$. The $\mathbb{F}$-algebra $\mathbb{F}^{\bar{N}}$ has its (finite) standard $\mathbb{F}$-basis

$$
\begin{gathered}
\epsilon_{\bar{i}}, \bar{i} \in \bar{N}, \epsilon_{\bar{i}}(\bar{t}):=\delta_{\bar{i}, \bar{t}}, \mathbb{F}^{\bar{N}}=\oplus_{\bar{i} \in \bar{N}} \mathbb{F} \epsilon_{\bar{i}}, \\
\epsilon_{\bar{i}} \epsilon_{\bar{j}}=\delta_{\bar{i}, \bar{j}} \epsilon_{\bar{i}}, \sum_{\bar{i} \in \bar{N}} \epsilon_{\bar{i}}=(1)=1_{\mathbb{F}^{N}} .
\end{gathered}
$$


The $\epsilon_{\bar{i}}$ are complete orthogonal idempotents of $\mathbb{F}^{\bar{N}}$. From (25) we infer

$$
\mathbf{A}=\mathbb{F}^{\bar{N}}[N ; \circ]=\oplus_{\bar{i} \in \bar{N}, j \in N} \mathbb{F} \epsilon_{\bar{i}} q^{j}, q^{j} \epsilon_{\bar{i}}=\left(j \circ \epsilon_{\bar{i}}\right) q^{j}=\epsilon_{\bar{i}-\bar{j}} q^{j}, q^{-j} \epsilon_{\overline{0}} q^{j}=\epsilon_{\bar{j}} .
$$

Remark 4.1. If $\mathbb{F}=\mathbb{C}$ is the field of complex numbers the functions in $\mathbb{C}^{\bar{N}}$ can be described by the discrete Fourier transform (DFT). Let $d:=\sharp(\bar{N})$ be the order of $\bar{N}$. Then there are nondegenerate symmetric biadditive resp. bimultiplicative forms $\bar{j} \bullet \bar{k}$ and $<\bar{j}, \bar{k}>$ :

$$
\begin{aligned}
& <-,->: \quad \bar{N} \times \bar{N} \stackrel{\bullet}{\rightarrow} \mathbb{Z} / \mathbb{Z} d \rightarrow S^{1}:=\{z \in \mathbb{C} ;|z|=1\}, \\
& (\bar{j}, \bar{k}) \quad \mapsto \bar{j} \bullet \bar{k} \mapsto<\bar{j}, \bar{k}>:=\exp (2 \pi i \bar{j} \bullet \bar{k} / d)
\end{aligned}
$$

With these data the DFT and the Fourier inversion formula are given by the inverse isomorphisms

$$
\begin{gathered}
\text { Four : } \mathbb{C}^{\bar{N}} \stackrel{\cong}{\longrightarrow} \mathbb{C}^{\bar{N}}, a \leftrightarrow \widehat{a}, \\
\widehat{a}(\bar{k}):=\sum_{j \in \bar{N}} a(\bar{j})<-\bar{j}, \bar{k}>, a(\bar{j})=d^{-1} \sum_{\bar{k} \in \bar{N}} \widehat{a}(\bar{k})<\bar{j}, \bar{k}> \\
\Longrightarrow \mathbb{C}^{\bar{N}}=\oplus_{\bar{j} \in \bar{N}} \mathbb{C}_{\bar{j}}=\oplus_{\bar{k} \in \bar{N}}=\mathbb{C}<-, \bar{k}>.
\end{gathered}
$$

In the simplest case one has

$$
N=\mathbb{Z} d \subset \mathbb{Z}, \bar{N}=\mathbb{Z} / \mathbb{Z} d, \bar{j} \bullet \bar{k}=\overline{j k},<\bar{j}, \bar{k}>=\exp (2 \pi i j k / d) .
$$

In other words, the $d$-periodic functions $a: \mathbb{Z} \rightarrow \mathbb{C}$ with $a(j)=a(j+d)$ for all $j \in \mathbb{Z}$ have the unique basis representation

$$
a(j)=d^{-1} \sum_{\bar{k} \in \mathbb{Z} / \mathbb{Z} d} \widehat{a}(\bar{k}) \exp (2 \pi i j k / d), \mathbb{C}^{\mathbb{Z} / \mathbb{Z} d}=\oplus_{\bar{k} \in \mathbb{Z} / \mathbb{Z} d} \mathbb{C} \exp (2 \pi i(-) k / d) .
$$

Equation (70) implies the equivalence

$$
\begin{aligned}
& \sum_{\bar{i} \in \bar{N}, j \in N} a(\bar{i}, j) \epsilon_{\bar{i}} q^{j} \in \operatorname{center}(\mathbf{A}) \\
& \Longleftrightarrow \forall \bar{k} \in \bar{N}, m \in N:\left(\sum_{\bar{i} \in \bar{N}, j \in N} a(\bar{i}, j) \epsilon_{\bar{i}} q^{j}\right) \epsilon_{\bar{k}} q^{m}=\epsilon_{\bar{k}} q^{m}\left(\sum_{\bar{i} \in \bar{N}, j \in N} a(\bar{i}, j) \epsilon_{\bar{i}} q^{j}\right) \\
& \Longleftrightarrow \forall \bar{k} \in \bar{N}, m \in N: \sum_{\bar{i} \in \bar{N}, j \in N} a(\bar{i}, j) \epsilon_{\bar{i}} \epsilon_{\bar{k}-\bar{j}} q^{j+m}=\sum_{\bar{i} \in \bar{N}, j \in N} a(\bar{i}, j) \epsilon_{\bar{k}} \epsilon_{\bar{i}-m} q^{j+m} \\
& \Longleftrightarrow \forall k, m, j \in N: \sum_{\bar{i} \in \bar{N}} a(\bar{i}, j) \delta_{\bar{i}, \bar{k}-\bar{j}} \epsilon_{\bar{i}}=\sum_{\bar{i} \in \bar{N}} a(\bar{i}, j) \delta_{\bar{i}, \bar{k}+\bar{m}} \epsilon_{\bar{k}} \\
& \Longleftrightarrow \forall k, m, j \in N: a(\bar{k}-\bar{j}, j) \epsilon_{\bar{k}-\bar{j}}=a(\bar{k}+\bar{m}, j) \epsilon_{\bar{k}} \quad \text { if } \bar{j} \neq \overline{0} \\
& \Longleftrightarrow \forall k, m, j \in N: a(\bar{k}-\bar{j}, j)=\left\{\begin{array}{l}
0 \quad \text { if } \bar{j}=\overline{0} \\
a(\bar{k}+\bar{m}, j)
\end{array}\right. \\
& \Longrightarrow \operatorname{center}(\mathbf{A})=\mathbf{Z}=\oplus_{j \in L} \mathbb{F}\left(\sum_{\bar{i} \in \bar{N}} \epsilon_{\bar{i}}\right) q^{j}=\oplus_{j \in L} \mathbb{F} q^{j} .
\end{aligned}
$$


Choose any section $\sigma: \bar{N} \rightarrow N$ of the canonical map $N \rightarrow N / L$ such that

$\overline{\sigma(\bar{i})}=\sigma(\bar{i})+L=\bar{i}$ and thus $L \times \bar{N} \rightarrow N=L+\sigma(\bar{N}),(t, \bar{j}) \mapsto t+\sigma(\bar{j})$,

is bijective. Then $\forall t+\sigma(\bar{j}) \in N=L+\sigma(\bar{N}): q^{t+\sigma(\bar{j})}=q^{t} q^{\sigma(\bar{j})}$

$\Longrightarrow \mathbf{A}=\oplus_{\bar{i}, \bar{j} \in \bar{N}, t \in L} \mathbb{F} \epsilon_{\bar{i}} q^{t} q^{\sigma(\bar{j})}=\oplus_{\bar{i}, \bar{j} \in \bar{N}} \mathbf{Z} \epsilon_{\bar{i}} q^{\sigma(\bar{j})}$

Further $\forall \bar{i} \in \bar{N}: q^{-\sigma(\bar{i})} \epsilon_{\overline{0}} q^{\sigma(\bar{i})}=\epsilon_{\bar{i}}$.

Hence $\mathbf{A}$ is a free $\mathbf{Z}$-module with the finite basis $\epsilon_{\bar{i}} q^{\sigma(\bar{j})}, \bar{i}, \bar{j} \in \bar{N}$. Since $\mathbf{Z}$ is noetherian so is $\mathbf{A}$ both as module over its center $\mathbf{Z}$ and as ring.

We consider the signal $\mathbb{F}$-module $W=\mathbb{F}^{N}$ that is also an A-module with the action

$$
\begin{gathered}
\left(\left(\sum_{j \in N} a_{j} q^{j}\right) \circ w\right)(t)=\sum_{j \in N} a_{j}(t) w(t+j), w \in W:=\mathbb{F}^{N}, t \in N, \\
\left(\sum_{\bar{i} \in \bar{N}, j \in N} a(\bar{i}, j) \epsilon_{\bar{i}} q^{j} \circ w\right)(t)=\sum_{\bar{i} \in \bar{N}, j \in N} a(\bar{i}, j) \delta_{\bar{i}, \bar{t}} w(t+j), a(\bar{i}, j) \in \mathbb{F} .
\end{gathered}
$$

The orthogonal idempotents $\epsilon_{\bar{i}}$ of $\mathbf{K}$ are also such in $\mathbf{A}$ and hence

$$
\mathbf{A}=\oplus_{\bar{j} \in \bar{N}} \mathbf{A} \epsilon_{\bar{j}} \text { with } \mathbf{A} \epsilon_{\bar{j}}=\mathbf{A} q^{-\sigma(\bar{j})} \epsilon_{\overline{0}} q^{\sigma(\bar{j})}=\mathbf{A} \epsilon_{\overline{0}} q^{\sigma(\bar{j})}, \mathbf{A} \epsilon_{\overline{0}} \cong \mathbf{A} \mathbf{A} \epsilon_{\bar{j}}, f \mapsto f q^{\sigma(\bar{j})} .
$$

Here we used that $q^{+/-\sigma(\bar{j})}$ are invertible. So the direct summands $\mathbf{A} \epsilon_{\bar{j}}$ of $\mathbf{A}$ are all isomorphic to $\mathbf{A} \epsilon_{\overline{0}}$. Since $\mathbf{A}$ is a progenerator of ${ }_{\mathbf{A}} \mathbf{M o d}[14$, p.54], i.e. a finitely generated projective module and generator, the cyclic module $P:=\mathbf{A} \epsilon_{0}$ is also a progenerator. Consider the $\mathbb{F}$-algebra $\mathbf{B}=\operatorname{Hom}_{\mathbf{A}}(P, P)$. It acts on $P$ from the left via $b x:=b(x)$ for $b \in \mathbf{B}$ and $x \in P$ and makes it an $(\mathbf{A}, \mathbf{B})$-bimodule since $a(b x)=$ $a b(x)=b(a x)$ for $a \in \mathbf{A}, b \in \mathbf{B}, x \in P$. Let $\mathbf{M o d}_{\mathbf{B}}$ be the category of B-right modules. The bimodule $P$ induces the Hom-functor

$$
\begin{gathered}
\operatorname{Hom}_{\mathbf{A}}(P,-):{ }_{\mathbf{A}} \operatorname{Mod} \rightarrow \operatorname{Mod}_{\mathbf{B}}, M \mapsto \operatorname{Hom}_{\mathbf{A}}(P, M) \text { with } \\
(\varphi b)(x):=\varphi(b x)=\varphi(b(x)), \varphi \in \operatorname{Hom}_{\mathbf{A}}(P, M), b \in \mathbf{B}=\operatorname{Hom}_{\mathbf{A}}(P, P), x \in P .
\end{gathered}
$$

According to the Morita equivalence theorems the following result holds:

Result 4.2. ( $c f .[14, \S 18 C, \S 18 D])$

1. The functor $\operatorname{Hom}_{\mathbf{A}}(P,-)$ is a categorical equivalence and, of course, exact.

2. The quasi-inverse functor of $\operatorname{Hom}_{\mathbf{A}}(P,-)$ is the functor

$$
\begin{gathered}
\operatorname{Mod}_{\mathbf{B}} \rightarrow{ }_{\mathbf{A}} \mathbf{M o d}, Y \mapsto Y \otimes_{\mathbf{B}} P \text { with } \\
a(y \otimes x):=y \otimes a x, a \in \mathbf{A}, y \in Y, x \in P, \text { especially } \\
\operatorname{Hom}_{\mathbf{A}}(P, \mathbf{A}) \otimes_{\mathbf{B}} P \cong \mathbf{A} \mathbf{A}, \varphi \otimes x \mapsto \varphi(x) .
\end{gathered}
$$

3. The canonical map $\mathbf{A} \rightarrow \operatorname{Hom}_{\mathbf{B}}(P, P), a \mapsto(x \mapsto a x)$, is a Z-algebra isomorphism. 
We modify and simplify Result 4.2 due to the special $P=\mathbf{A} \epsilon_{\overline{0}}$ : There is the standard algebra antiisomorphism

$$
\begin{gathered}
\Phi: \mathbf{B}=\operatorname{Hom}_{\mathbf{A}}\left(\mathbf{A} \epsilon_{\overline{0}}, \mathbf{A} \epsilon_{\overline{0}}\right) \cong \epsilon_{\text {anti }} \epsilon_{\overline{0}} \mathbf{A} \epsilon_{\overline{0}}, b \mapsto b\left(\epsilon_{\overline{0}}\right), \text { since } \\
\forall b \in \mathbf{B}: b\left(\epsilon_{\overline{0}}\right)=b\left(\epsilon_{\overline{0}}\right) \epsilon_{\overline{0}}=b\left(\epsilon_{\overline{0}}^{2}\right)=\epsilon_{\overline{0}} b\left(\epsilon_{\overline{0}}\right) \in \epsilon_{\overline{0}} \mathbf{A} \epsilon_{\overline{0}}, \\
\forall b_{1}, b_{2} \in \mathbf{B}:\left(b_{1} b_{2}\right)\left(\epsilon_{\overline{0}}\right)=b_{1}\left(b_{2}\left(\epsilon_{\overline{0}}\right) \epsilon_{\overline{0}}\right)=b_{2}\left(\epsilon_{\overline{0}}\right) b_{1}\left(\epsilon_{\overline{0}}\right) .
\end{gathered}
$$

We compute $\epsilon_{\overline{0}} \mathbf{A}, \mathbf{A} \epsilon_{\overline{0}}$ and $\epsilon_{\overline{0}} \mathbf{A} \epsilon_{\overline{0}}$ as free $\mathbf{Z}$-modules:

$$
\begin{aligned}
& \epsilon_{\bar{i}} q^{j} \epsilon_{\overline{0}}=\epsilon_{\bar{i}} \epsilon_{-\bar{j}} q^{j}=\delta_{\bar{i},-\bar{j}} \epsilon_{\bar{i}} q^{j}, \epsilon_{\overline{0}} \epsilon_{\bar{i}} q^{j} \epsilon_{\overline{0}}=\delta_{\bar{i},-\bar{j}} \epsilon_{\overline{0}} \epsilon_{\bar{i}} q^{j}=\delta_{\overline{0}, \bar{i}} \delta_{\overline{0}, \bar{j}} \epsilon_{\overline{0}} q^{j} \Longrightarrow \\
& \epsilon_{\overline{0}} \mathbf{A}=\epsilon_{\overline{0}}\left(\oplus_{\bar{i} \in \bar{N}, j \in N} \mathbb{F} \epsilon_{\bar{i}} q^{j}\right)=\oplus_{j \in N} \mathbb{F} \epsilon_{\overline{0}} q^{j}=\oplus_{j \in \bar{N}} \mathbf{Z} \epsilon_{\overline{0}} q^{\sigma(\bar{j})} \\
& \mathbf{A} \epsilon_{\overline{0}}=\left(\oplus_{\bar{i} \in \bar{N}, j \in N} \mathbb{F} \epsilon_{\bar{i}} q^{j}\right) \epsilon_{\overline{0}}=\oplus_{j \in N} \mathbb{F} \epsilon_{-\bar{j}} q^{j}=\oplus_{\bar{j} \in \bar{N}} \mathbf{Z} \epsilon_{-\bar{j}} q^{\sigma(\bar{j})}, \\
& \epsilon_{\overline{0}} \mathbf{A} \epsilon_{\overline{0}}=\epsilon_{\overline{0}}\left(\oplus_{\bar{i} \in \bar{N}, j \in N} \mathbb{F} \epsilon_{\bar{i}} q^{j}\right) \epsilon_{\overline{0}}=\oplus_{j \in L} \mathbb{F} \epsilon_{\overline{0}} q^{j}=\oplus_{j \in L} \mathbb{F} q^{j} \epsilon_{\overline{0}}=\mathbf{Z} \epsilon_{\overline{0}} \cong \mathbf{Z} .
\end{aligned}
$$

Since $\mathbf{Z}$ is commutative the antiisomorphism $\Phi$ is indeed a ring isomorphism and left and right modules over

$$
\mathbf{B} \cong \epsilon_{\overline{0}} \mathbf{A} \epsilon_{\overline{0}}=\mathbf{Z} \epsilon_{\overline{0}} \cong \operatorname{center}(\mathbf{A})=\mathbf{Z}
$$

coincide. In particular, $\operatorname{Mod}_{\mathbf{B}}={ }_{\mathbf{Z}} \operatorname{Mod}$ and $\mathbf{A} \epsilon_{\overline{0}}$ is an $(\mathbf{A}, \mathbf{Z})$-bimodule with the multiplications

$$
a_{1}\left(a_{2} \epsilon_{\overline{0}}\right)=\left(a_{1} a_{2}\right) \epsilon_{\overline{0}} \text { and }\left(a_{2} \epsilon_{\overline{0}}\right) f=\left(a_{2} \epsilon_{\overline{0}}\right)\left(f \epsilon_{\overline{0}}\right)=f a_{2} \epsilon_{\overline{0}}, a_{1}, a_{2} \in \mathbf{A}, f \in \mathbf{Z} .
$$

For every $M \in \mathbf{A}_{\mathbf{A}} \mathbf{M o d}$ we get the $\mathbf{Z}$-isomorphism

$$
\operatorname{Hom}_{\mathbf{A}}\left(\mathbf{A} \epsilon_{\overline{0}}, M\right) \underset{\mathbf{Z}}{\cong} \epsilon_{\overline{0}} M, \varphi \mapsto \varphi\left(\epsilon_{\overline{0}}\right) .
$$

With the preceding modifications Result 4.2 obtains the following form:

\section{Corollary 4.3. 1. The functor}

$$
\text { AMod } \rightarrow{ }_{\mathbf{z}} \text { Mod, } M \mapsto \epsilon_{\overline{0}} M,
$$

is a categorical equivalence, in particular exact and for $M_{1}, M_{2} \in \mathbf{A}$ Mod there is the $\mathbf{Z}$-isomorphism

$$
\operatorname{Hom}_{\mathbf{A}}\left(M_{1}, M_{2}\right) \cong \operatorname{Hom}_{\mathbf{Z}}\left(\epsilon_{\overline{0}} M_{1}, \epsilon_{\overline{0}} M_{2}\right), G \mapsto G \mid \epsilon_{\overline{0}} M_{1} .
$$

2. The quasi-inverse functor of $M \mapsto \epsilon_{\overline{0}} M$ is the functor

$$
\mathbf{z} \operatorname{Mod} \rightarrow \mathbf{A}_{\mathbf{A}} \operatorname{Mod}, Y \mapsto \mathbf{A} \epsilon_{\overline{0}} \otimes \mathbf{z} Y
$$

3. The canonical map

$$
\Theta: \mathbf{A} \rightarrow \operatorname{Hom}_{\mathbf{Z}}\left(\mathbf{A} \epsilon_{\overline{0}}, \mathbf{A} \epsilon_{\overline{0}}\right) \text {, with } \Theta\left(a_{1}\right)\left(a_{2} \epsilon_{\overline{0}}\right):=a_{1} a_{2} \epsilon_{\overline{0}},
$$

is a Z-algebra isomorphism.

Since $\mathbf{A} \epsilon_{\overline{0}}$ has the $\mathbf{Z}$-basis $\epsilon_{-\bar{k}} q^{\sigma(\bar{k})}, \bar{k} \in \bar{N}$, the $\mathbf{Z}$-algebras in (85) are isomorphic to the matrix algebra $\mathbf{Z}^{\bar{N} \times \bar{N}}$. 


\subsection{Stacked modules and behaviors}

For the A-signal module $W=\mathbb{F}^{N}$ we compute $\epsilon_{\overline{0}} \circ W$ by

$$
\begin{gathered}
\forall w \in W \forall t \in N:\left(\epsilon_{\overline{0}} \circ w\right)(t)=\epsilon_{\overline{0}}(\bar{t}) w(t)=\delta_{\overline{0}, \bar{t}} w(t) \Longrightarrow \\
\epsilon_{\overline{0}} \circ W=\left\{w \in \mathbb{F}^{N} ; w \mid(N \backslash L)=0\right\} \cong \mathbb{F}^{L}, w \leftrightarrow \widehat{w}, \\
\text { where } w(t)= \begin{cases}\widehat{w}(t) & \text { if } \bar{t}=\overline{0} \text { or } t \in L \\
0 & \text { if } \bar{t} \neq \overline{0} \text { or } t \in N \backslash L\end{cases}
\end{gathered}
$$

Identify $\epsilon_{\overline{0}} \circ \mathbb{F}^{N}=\mathbb{F}^{L} \subseteq \mathbb{F}^{N}, w=\widehat{w}$, and define $\widehat{W}=\mathbb{F}^{L} \subset W=\mathbb{F}^{N}$.

This identification signifies that $\widehat{w} \in \mathbb{F}^{L}$ is extended to $w \in \mathbb{F}^{N}$ by $w \mid(N \backslash L)=0$. Then $\widehat{W}$ is a $\mathbf{Z}$-submodule of $W$ since

$$
\forall i \in L:\left(q^{i} \circ \widehat{w}\right)(t)=\left\{\begin{array}{ll}
\widehat{w}(t+i) & \text { if } t \in L \\
0 & \text { if } t, i+t \in N \backslash L
\end{array} .\right.
$$

Theorem 4.4. With the identification from (86) the signal modules $\mathbb{A}^{N}$ and $\mathbb{Z}^{L}$ are related by $\mathbb{F}^{L}=\epsilon_{\overline{0}} \circ \mathbb{F}^{N}$. Since $\mathbb{Z}^{L}$ is an injective cogenerator due to (20) or [15] the categorical equivalence from (82) implies that $\mathbb{A}^{N}$ is also an injective cogenerator.

For any $M \in{ }_{\mathbf{A}} \mathbf{M o d}$ the equivalence ${ }_{\mathbf{A}} \operatorname{Mod} \rightarrow{ }_{\mathbf{z}} \operatorname{Mod}, M \mapsto \epsilon_{\overline{0}} M$, furnishes

$$
\begin{array}{ccc}
\operatorname{Hom}_{\mathbf{A}}\left(M, \mathbb{F}^{N}\right) \cong \operatorname{Hom}_{\mathbf{Z}}\left(\epsilon_{\overline{0}} M, \epsilon_{\overline{0}} \circ \mathbb{F}^{N}\right) & \cong & \operatorname{Hom}_{\mathbf{Z}}\left(\epsilon_{\overline{0}} M, \mathbb{F}^{L}\right) \\
\Phi & \longleftrightarrow \mid \epsilon_{\overline{0}} M
\end{array}
$$

For $x \in M, \bar{j} \in \bar{N}, w:=\Phi(x), \widehat{w}_{\bar{j}}:=\Phi\left(\epsilon_{\overline{0}} q^{\sigma(\bar{j})} x\right)=\epsilon_{\overline{0}} q^{\sigma(\bar{j})} \circ w$ this implies

$$
\begin{gathered}
\forall t \in L \forall \bar{j} \in \bar{N}: \widehat{w}_{\bar{j}}(t)=w(t+\sigma(\bar{j})), \epsilon_{\bar{j}} \circ w \underset{(\overline{72})}{=} q^{-\sigma(\bar{j})} \circ \widehat{w}_{\bar{j}}, \\
w=\sum_{\bar{j} \in \bar{N}} \epsilon_{\bar{j}} \circ w=\sum_{\bar{j} \in \bar{N}} q^{-\sigma(\bar{j})} \circ \widehat{w}_{\bar{j}} .
\end{gathered}
$$

Hence $\widehat{w}_{\bar{j}} \in \widehat{W}=\mathbb{F}^{L}$ is given by and determines the values of $w$ on the residue class $\bar{j}=\sigma(\bar{j})+L$. Equation (89) also holds if for a finite index set $\Lambda$ the modules $\mathbf{A}, \mathbb{F}^{N}$ resp. $\mathbb{F}^{L}$ are replaced by $\mathbf{A}^{\Lambda},\left(\mathbb{F}^{N}\right)^{\Lambda}$ resp. $\left(\mathbb{F}^{\Lambda}\right)^{L}$.

In the sequel we use the notations from (30) and (34) for finite index sets $\Lambda$ and $\Gamma$. Assume that $\mathbf{w}=\left(\mathbf{w}_{\lambda}\right)_{\lambda \in \Lambda} \in M^{\Lambda}=M^{(\Lambda)}$ is a finite family of generators of $M$, i.e., $M=\sum_{\lambda \in \Lambda} \mathbf{A} \mathbf{w}_{\lambda}$. Consider the free $\mathbf{A}$-module $\mathbf{A}^{\Lambda}=\mathbf{A}^{(\Lambda)}$ with the elements $\xi=(\xi(\lambda))_{\lambda \in \Lambda}: \Lambda \rightarrow \mathbf{A}$ and the standard basis $\delta_{\lambda}, \lambda \in \Lambda$, with $\delta_{\lambda}(\mu)=\delta_{\lambda, \mu}$ and $\xi=\sum_{\lambda \in \Lambda} \xi(\lambda) \delta_{\lambda}$. Define the canonical map

$$
\begin{gathered}
\operatorname{can}_{\mathbf{w}}: \mathbf{A}^{\Lambda} \rightarrow M, \delta_{\lambda} \mapsto \mathbf{w}_{\lambda}, \xi \mapsto \xi \mathbf{w}:=\sum_{\lambda \in \Lambda} \xi(\lambda) \mathbf{w}_{\lambda}, \\
U:=\operatorname{ker}\left(\operatorname{can}_{\mathbf{w}}\right)=\left\{\xi \in \mathbf{A}^{\Lambda} ; \xi \mathbf{w}=0\right\} \Longrightarrow \mathbf{A}^{\Lambda} / U \underset{\text { ident. }}{=} M, \xi+U=\xi \mathbf{w} .
\end{gathered}
$$

With the identifications from (34) we get

$$
\begin{gathered}
W^{\Lambda}=\left(\mathbb{F}^{N}\right)^{\Lambda}=\mathbb{F}^{N \times \Lambda}=\left(\mathbb{F}^{\Lambda}\right)^{N} \ni w \text { with } \\
w(\lambda)(t)=w(\lambda, t)=w(t)(\lambda) \text { for } \lambda \in \Lambda, t \in N .
\end{gathered}
$$


There results the $\mathbf{Z}$-linear isomorphism (cf. (17))

$$
\begin{gathered}
\operatorname{Hom}_{\mathbf{A}}(M, W) \underset{\text { Malgrange } 1962}{\cong} \mathcal{B}:=U^{\perp}:=\left\{w \in W^{\Lambda} ; \forall \xi \in U: \xi \circ w=0\right\}, \\
\Phi \longleftrightarrow w, w(\lambda):=\Phi\left(\mathbf{w}_{\lambda}\right), \Phi(\xi \mathbf{w})=\xi \circ w:=\sum_{\lambda \in \Lambda} \xi(\lambda) \circ w(\lambda) .
\end{gathered}
$$

The $\mathbf{Z}$-module $\mathcal{B}$ is the $\mathbf{A} W$-behavior associated to the equation module $U$ or the system module $M$. Consider two f.g. A-modules $\mathbf{A}^{\Lambda_{i}} / U_{i}, i=1,2$, with their associated behaviors $\mathcal{B}_{i}:=U_{i}^{\perp} \subseteq W^{\Lambda_{i}}$. Define the Z-module $\operatorname{Hom}\left(\mathcal{B}_{2}, \mathcal{B}_{1}\right)$ of behavior morphisms as

$$
\operatorname{Hom}\left(\mathcal{B}_{2}, \mathcal{B}_{1}\right):=\left\{\Phi \in \operatorname{Hom}_{\mathbf{Z}}\left(\mathcal{B}_{2}, \mathcal{B}_{1}\right) ; \exists P \in \mathbf{A}^{\Lambda_{1} \times \Lambda_{2}} \text { with }(\star)\right\} \text { where }
$$

$(\star): \forall w_{2} \in \mathcal{B}_{2}, w_{1}:=\Phi\left(w_{2}\right), \forall \lambda_{1} \in \Lambda_{1}: w_{1}\left(\lambda_{1}\right)=\sum_{\lambda_{2} \in \Lambda_{2}} P\left(\lambda_{1}, \lambda_{2}\right) \circ w_{2}\left(\lambda_{2}\right)$.

Since ${ }_{\mathbf{A}} W$ is an injective cogenerator standard algebraic arguments imply

Corollary 4.5. (cf. [5, Cor. 2.6]) There are canonical Z-isomorphisms

$$
\begin{gathered}
\left\{P \in \mathbf{A}^{\Lambda_{1} \times \Lambda_{2}} ; U_{1} P \subseteq U_{2}\right\} /\left\{P \in \mathbf{A}^{\Lambda_{1} \times \Lambda_{2}} ; \mathbf{A}^{\Lambda_{1}} P \subseteq U_{2}\right\} \\
\cong \operatorname{Hom}_{\mathbf{A}}\left(M_{1}, M_{2}\right) \cong \operatorname{Hom}\left(\mathcal{B}_{2}, \mathcal{B}_{1}\right), \bar{P} \mapsto(\circ P)_{\text {ind }} \mapsto P \circ \text { where } \\
\bar{P}=P+\left\{P \in \mathbf{A}^{\Lambda_{1} \times \Lambda_{2}} ; \mathbf{A}^{\Lambda_{1}} P \subseteq U_{2}\right\},(\circ P)_{\text {ind }}\left(\xi_{1}+U_{1}\right):=\xi_{1} P+U_{2} .
\end{gathered}
$$

In other words, the functor $M=\mathbf{A}^{\Lambda} / U \mapsto \mathcal{B}:=U^{\perp}$ is a duality or contravariant equivalence from the category of $\mathbf{A}$-modules with a given finite family of generators and the category of $\mathbf{A} W$-behaviors.

From

$$
\mathbf{A}=\sum_{\bar{i}, \bar{j} \in \bar{N}} \mathbf{Z} \epsilon_{\bar{i}} q^{\sigma(j)} \text { we infer } \epsilon_{\overline{0}} \mathbf{A}^{\Lambda}=\oplus_{(\bar{j}, \lambda) \in \bar{N} \times \Lambda} \mathbf{Z} \epsilon_{\overline{0}} q^{\sigma(\bar{j})} \delta_{\lambda} .
$$

We identify this free $\mathbf{Z}$-module with $\mathbf{Z}^{\bar{N} \times \Lambda}$ via

$$
\epsilon_{\overline{0}} \mathbf{A}^{\Lambda}=\mathbf{Z}^{\bar{N} \times \Lambda}, \delta_{(\bar{j}, \lambda)}=\epsilon_{\overline{0}} q^{\sigma(\bar{j})} \delta_{\lambda} .
$$

The exact sequence of A-modules

$$
\begin{gathered}
0 \rightarrow U \stackrel{\text { inj }}{\longrightarrow} \mathbf{A}^{\Lambda} \stackrel{\operatorname{can}_{W}}{\longrightarrow} M \rightarrow 0 \text { induces the exact sequence of Z-modules } \\
0 \rightarrow \epsilon_{\overline{0}} U \stackrel{\text { inj }}{\longrightarrow} \epsilon_{\overline{0}} \mathbf{A}^{\Lambda}=\oplus_{(\bar{j}, \lambda) \in \bar{N} \times \Lambda} \mathbf{Z} \epsilon_{\overline{0}} q^{\sigma(\bar{j})} \delta_{\lambda} \stackrel{\text { can }_{\mathbf{w}} \mid \epsilon_{\overline{0}} \mathbf{A}^{\Lambda}}{\longrightarrow} \epsilon_{\overline{0}} M \rightarrow 0
\end{gathered}
$$

with $\widehat{\mathbf{w}}_{\bar{j}, \lambda}:=\operatorname{can}_{\mathbf{w}}\left(\epsilon_{\overline{0}} q^{\sigma(\bar{j})} \delta_{\lambda}\right)=\epsilon_{\overline{0}} q^{\sigma(\bar{j})} \mathbf{w}_{\lambda}, \widehat{\mathbf{w}}=\left(\widehat{\mathbf{w}}_{\bar{j}, \lambda}\right)_{(\bar{j}, \lambda) \in \bar{N} \times \Lambda} \in \epsilon_{\overline{0}} M^{\bar{N} \times \Lambda}$,

$$
\text { hence } \epsilon_{\overline{0}} M=\sum_{(\bar{j}, \lambda) \in \bar{N} \times \Lambda} \mathbf{Z} \widehat{\mathbf{w}}_{\bar{j}, \lambda} \text {. }
$$

We define

$$
\begin{gathered}
\widehat{U}:=\epsilon_{\overline{0}} U \subseteq \epsilon_{\overline{0}} \mathbf{A}^{\Lambda}=\mathbf{Z}^{\bar{N} \times \Lambda} \text { and identify } \\
\epsilon_{\overline{0}} \mathbf{A}^{\Lambda} / \epsilon_{\overline{0}} U=\mathbf{Z}^{\bar{N} \times \Lambda} / \widehat{U}=\epsilon_{\overline{0}} M, \epsilon_{\overline{0}} q^{\sigma(\bar{j})} \delta_{\lambda}+\widehat{U}=\widehat{\mathbf{w}}_{\bar{j}, \lambda} .
\end{gathered}
$$


As in (91) we also identify

$$
\begin{aligned}
\widehat{W}^{\bar{N} \times \Lambda} & =\left(\mathbb{F}^{L}\right)^{\bar{N} \times \Lambda}=\mathbb{F}^{L \times \bar{N} \times \Lambda}=\left(\mathbb{F}^{\bar{N} \times \Lambda}\right)^{L} \ni \widehat{w} \text { with } \\
\widehat{w}(\bar{j}, \lambda)(t) & =\widehat{w}(t, \bar{j}, \lambda)=\widehat{w}(t)(\bar{j}, \lambda) \text { for } t \in L, \bar{j} \in \bar{N}, \lambda \in \Lambda .
\end{aligned}
$$

In this case the Malgrange isomorphism (cf. (17), (92)) furnishes the $\mathbf{Z}$-isomorphism

$$
\begin{gathered}
\operatorname{Hom}_{\mathbf{Z}}\left(\epsilon_{\overline{0}} M, \widehat{W}\right) \cong \widehat{\mathcal{B}}:=\widehat{U}^{\perp}:= \\
\left\{\widehat{w} \in\left(\mathbb{F}^{L}\right)^{\bar{N} \times \Lambda} ; \forall \eta \in \widehat{U}\left(\subseteq \mathbf{Z}^{\bar{N} \times \Lambda}\right): \eta \circ \widehat{w}=\sum_{(\bar{j}, \lambda) \in \bar{N} \times \lambda} \eta(\bar{j}, \lambda) \circ \widehat{w}(\bar{j}, \lambda)=0\right\}, \\
\varphi \mapsto \widehat{w}, \varphi\left(\widehat{\mathbf{w}}_{\bar{j}, \lambda}\right)=\widehat{w}(\bar{j}, \lambda) .
\end{gathered}
$$

The $\mathbf{Z}$-module $\widehat{\mathcal{B}}$ is the $\mathbf{z} \widehat{W}$-behavior associated to the equation module $\widehat{U}$ and the system module $\epsilon_{\overline{0}} M$. Since $\mathbf{z} \widehat{W}$ is an injective cogenerator due to (20) or [15] the assignment $Y \mapsto \operatorname{Hom}_{\mathbf{Z}}(Y, \widehat{W})$ establishes the standard duality between finitely generated modules over the Laurent polynomial algebra $\mathbf{Z}=\mathbb{F}[L]=\mathbb{F}\left[x_{1}, x_{1}^{-1}, \cdots, x_{r}, x_{r}^{-1}\right]$ and the associated $\mathbf{z} \widehat{W}$-behaviors.

Theorem and Definition 4.6. For the data from (90)-(100) there is the commutative diagram of $\mathbf{Z}$-isomorphisms

$$
\begin{aligned}
& \operatorname{Hom}_{\mathbf{A}}(M, W) \stackrel{\cong}{\longrightarrow} \operatorname{Hom}_{\mathbf{Z}}\left(\epsilon_{\overline{0}} M, \widehat{W}\right), \quad \Phi \mapsto \Phi \mid \epsilon_{\overline{0}} M \\
& \downarrow \cong \downarrow \cong \downarrow \downarrow \downarrow \\
& \mathcal{B} \quad \cong \quad \widehat{\mathcal{B}}, \quad w \mapsto \quad \widehat{w} \text {, where } \\
& \bigcap_{\mathbb{F}^{N \times \Lambda}} \cong \mathbb{F}^{L \times \bar{N} \times \Lambda} \\
& w(\lambda)=\Phi\left(\mathbf{w}_{\lambda}\right), \widehat{w}(\bar{j}, \lambda)=\Phi\left(\epsilon_{\overline{0}} q^{\sigma(\bar{j})} \mathbf{w}_{\lambda}\right), q^{-\sigma(\bar{j})} \circ \widehat{w}(\bar{j}, \lambda)=\epsilon_{\bar{j}} \circ w(\lambda), \\
& \forall t \in L \forall \bar{j} \in \bar{N} \forall \lambda \in \Lambda: \widehat{w}(\bar{j}, \lambda)(t)=w(\lambda)(t+\sigma(\bar{j})), \\
& \forall \lambda \in \Lambda: w(\lambda)=\sum_{\bar{j} \in \bar{N}} \epsilon_{\bar{j}} \circ w(\lambda)=\sum_{\bar{j} \in \bar{N}} q^{-\sigma(\bar{j})} \circ \widehat{w}(\bar{j}, \lambda) \text {. }
\end{aligned}
$$

So the values $\widehat{w}(\bar{j}, \lambda)(t), t \in L$, coincide with the values of $w(\lambda)$ on the residue class $\bar{j}=L+\sigma(\bar{j})$. Therefore $w$ and $\widehat{w}$ resp. the $\mathbb{A}_{\mathbf{F}}{ }^{N}$-behavior $\mathcal{B}$ and the $\mathbf{Z}^{L}$-behavior $\widehat{\mathcal{B}}$ coincide up to a reordering of the components of the trajectories. The module $\epsilon_{\overline{0}} M$ resp. $\widehat{\mathcal{B}}$ are called the stacked form of $M$ resp. $\mathcal{B}$.

Proof. The commutativity of the diagram is a consequence of (89). The upper horizontal isomorphism follows from (88). Since the vertical maps are the Malgrange isomorphisms also the lower horizontal map is an isomorphism.

In other words, the behaviors defined by linear partial difference equations with $p e$ riodic coefficients are fully reduced to well-studied multidimensional behaviors defined by such equations with constant coefficients. In Section 5 we explain the connection of our theory with the work of our predecessors in the one-dimensional case.

We finally derive a kernel representation of $\widehat{\mathcal{B}}$ from a given one for $\mathcal{B}$ in (101): Since $\mathbf{A}$ 
is noetherian the submodule $U$ is finitely generated. Therefore assume that $U$ is given for finite $\Gamma$ and $R \in \mathbf{A}^{\Gamma \times \Lambda}$ as

$$
\begin{aligned}
& U=\mathbf{A}^{\Gamma} R=\sum_{\gamma \in \Gamma} \mathbf{A} R(\gamma,-), R(\gamma,-)=\sum_{\lambda \in \Lambda} R(\gamma, \lambda) \delta_{\lambda} \subseteq \mathbf{A}^{\Lambda}, R \in \mathbf{A}^{\Gamma \times \Lambda}, \\
& \text { hence } \mathcal{B}=\left\{w \in W^{\Lambda} ; R \circ w=0 \text { or } \forall \gamma \in \Gamma: \sum_{\lambda \in \Lambda} R(\gamma, \lambda) \circ w(\lambda)=0\right\} .
\end{aligned}
$$

We write $R(\gamma, \lambda)$ as

$$
\begin{gathered}
R(\gamma, \lambda)=\sum_{\bar{k}, \bar{\ell} \in \bar{N}} R((\bar{k}, \gamma),(\bar{\ell}, \lambda)) \epsilon_{\bar{k}} q^{\sigma(\bar{\ell})} \in \mathbf{A}:=\oplus_{\bar{k}, \bar{\ell} \in \bar{N}} \mathbf{Z} \epsilon_{\bar{k}} q^{\sigma(\bar{\ell})} \text { and } \\
t(\bar{j}, \bar{\ell}):{ }_{(\overline{72})} \sigma(\bar{j})+\sigma(\bar{\ell})-\sigma(\bar{j}+\bar{\ell}) \in L, \sigma(\bar{j})+\sigma(\bar{\ell})=\sigma(\bar{j}+\bar{\ell})+t(\bar{j}, \bar{\ell})
\end{gathered}
$$

and compute the $\mathbf{Z}$-generators of $\mathbf{Z}$-submodule $\widehat{U}=\epsilon_{\overline{0}} U$ by

$$
\begin{aligned}
& \epsilon_{\overline{0}} U=\sum_{\gamma \in \Gamma} \epsilon_{\overline{0}} \mathbf{A} R(\gamma,-)=\sum_{\gamma \in \Gamma} \epsilon_{\overline{0}} \mathbf{A} \sum_{\lambda \in \Lambda} R(\gamma, \lambda) \delta_{\lambda} \\
& =\left(\sum_{\gamma \in \Gamma, \bar{i}, \bar{j} \in \bar{N}} \mathbb{F}[L] \epsilon_{\overline{0}} \epsilon_{\bar{i}} q^{\sigma(\bar{j})}\right)\left(\sum_{\lambda \in \Lambda, \bar{k}, \bar{\ell} \in \bar{N}} R((\bar{k}, \gamma),(\bar{\ell}, \lambda)) \epsilon_{\bar{k}} q^{\sigma(\bar{\ell})} \delta_{\lambda}\right) \\
& =\sum_{\gamma, \bar{j}} \mathbb{F}[L] \sum_{\lambda, \bar{k}, \bar{\ell}} R((\bar{k}, \gamma),(\bar{\ell}, \lambda)) \epsilon_{\overline{0}} \epsilon_{\bar{k}-\bar{j}} q^{\sigma(\bar{j})+\sigma(\bar{\ell})} \delta_{\lambda} \\
& =\sum_{\gamma, \bar{j}} \mathbb{F}[L] \sum_{\lambda, \bar{\ell}} R((\bar{j}, \gamma),(\bar{\ell}, \lambda)) \epsilon_{\overline{0}} q^{t(\bar{j}, \bar{\ell})} q^{\sigma(\bar{j}+\bar{\ell})} \delta_{\lambda} \\
& =\sum_{\bar{j}+\overline{\bar{\ell}}=\bar{m}} \sum_{\bar{j}, \gamma} \mathbb{F}[L] \sum_{\lambda, \bar{m}} R((\bar{j}, \gamma),(\bar{m}-\bar{j}, \lambda)) q^{t(\bar{j}, \bar{m}-\bar{j})} \epsilon_{\overline{0}} q^{\sigma(\bar{m})} \delta_{\lambda} \\
& \Longrightarrow \widehat{U}=\epsilon_{\overline{0}} U=\sum_{(\bar{j}, \gamma) \in \bar{N} \times \Gamma} \mathbb{F}[L] \sum_{(\bar{m}, \lambda) \in \bar{N} \times \Lambda} \widehat{R}((\bar{j}, \gamma),(\bar{m}, \lambda)) \epsilon_{\overline{0}} q^{\sigma(\bar{m})} \delta_{\lambda}
\end{aligned}
$$

where $\forall(\bar{j}, \gamma) \in \bar{N} \times \Gamma,(\bar{m}, \lambda) \in \bar{N} \times \Lambda$ :

$\widehat{R}((\bar{j}, \gamma),(\bar{m}, \lambda)):=R((\bar{j}, \gamma),(\bar{m}-\bar{j}, \lambda)) q^{t(\bar{j}, \bar{m}-\bar{j})}, \widehat{R} \in \mathbf{Z}^{(\bar{N} \times \Gamma) \times(\bar{N} \times \Lambda)}$.

Theorem 4.7. In the Z-basis

$$
\delta_{(\bar{m}, \lambda)}=\epsilon_{0} q^{\sigma(\bar{m})} \delta_{\lambda} \in \epsilon_{\overline{0}} \mathbf{A}^{\Lambda} \underset{(96)}{=} \mathbf{Z}^{\bar{N} \times \Lambda},(\bar{m}, \lambda) \in \bar{N} \times \Lambda,
$$

of $\epsilon_{\overline{0}} \mathbf{A}^{\Lambda}$ the submodule $\widehat{U}=\epsilon_{\overline{0}} U$ is generated by the family

$$
\sum_{(\bar{m}, \lambda) \in \bar{N} \times \Lambda} \widehat{R}((\bar{j}, \gamma),(\bar{m}, \lambda)) \delta_{(\bar{m}, \lambda)},(\bar{j}, \gamma) \in \bar{N} \times \Gamma
$$

where $R$ and t are taken from (103) and $\widehat{R}$ from (104). For the $\mathbf{A} \mathbb{F}^{N}$-behavior $\mathcal{B}$ and 
the $\mathbf{Z}^{\mathbb{F}}$-behavior $\widehat{\mathcal{B}}$ this implies the kernel representations

$$
\begin{gathered}
\mathcal{B}=\left\{w \in\left(\mathbb{F}^{N}\right)^{\Lambda} ; \forall \gamma \in \Gamma: \sum_{\lambda \in \Lambda} R(\gamma, \lambda) \circ w(\lambda)=0\right\} \cong \widehat{\mathcal{B}}= \\
\left\{\widehat{w} \in\left(\mathbb{F}^{L}\right)^{\bar{N} \times \Lambda} ; \forall(\bar{j}, \gamma) \in \bar{N} \times \Gamma: \sum_{(\bar{m}, \lambda) \in \bar{N} \times \Lambda} \widehat{R}((\bar{j}, \gamma),(\bar{m}, \lambda)) \circ \widehat{w}(\bar{m}, \lambda)=0\right\} .
\end{gathered}
$$

In the following fashion the behavior $\widehat{\mathcal{B}}$ is canonically an ${ }_{\mathbf{A}} W$-behavior. Assume, more generally, any finite presentation or exact sequence of $\mathbf{Z}$-modules

$$
\begin{aligned}
& \begin{array}{ccccc}
\mathbf{Z}^{\Theta} & \stackrel{\circ S}{\longrightarrow} & \mathbf{Z}^{\Pi} & \longrightarrow & \epsilon_{\overline{0}} M \\
\eta & \mapsto & \eta S, \xi & \mapsto \mathbf{v}=\sum_{\pi \in \Pi} \xi(\pi) \mathbf{v}_{\pi} & \longrightarrow 0
\end{array} \\
& S \in \mathbf{Z}^{\Theta \times \Pi}, \epsilon_{\overline{0}} M=\sum_{\pi \in \Pi} \mathbf{Z}_{\mathbf{v}_{\pi}}, \epsilon_{\overline{0}} \mathbf{v}=\mathbf{v}:=\left(\mathbf{v}_{\pi}\right)_{\pi \in \Pi} \in \epsilon_{\overline{0}} M^{\Pi},
\end{aligned}
$$

and the associated $\mathrm{z} \widehat{W}$-behavior

$$
\widehat{\mathcal{C}}:=\left\{\widehat{v} \in \widehat{W}^{\Pi} ; S \circ \widehat{v}=0\right\} \cong \operatorname{Hom}_{\mathbf{Z}}\left(\epsilon_{\overline{0}} M, \widehat{W}\right) .
$$

The Morita equivalence induces the exact sequences of A-modules

$$
\begin{array}{ccccc}
\mathbf{A}^{\Theta} \epsilon_{\overline{0}} & \stackrel{\circ S}{\longrightarrow} & \mathbf{A}^{\Pi} \epsilon_{\overline{0}} & \longrightarrow & M \\
\eta \epsilon_{\overline{0}} & \mapsto & \eta \epsilon_{\overline{0}} S=\eta S \epsilon_{\overline{0}}, \xi \epsilon_{\overline{0}} & \mapsto & \xi \epsilon_{\overline{0}} \mathbf{v}=\xi \mathbf{v}
\end{array} \quad \longrightarrow 0 .
$$

With

$$
\operatorname{Hom}_{\mathbf{A}}\left(\mathbf{A} \epsilon_{\overline{0}}, W\right) \cong \epsilon_{\overline{0}} W=\left\{v \in W ;\left(1-\epsilon_{\overline{0}}\right) \circ v=0\right\}=\widehat{W}
$$

the exact sequence (110) implies the $\mathbf{A}_{\mathbf{A}} W$ - behavior

$$
\mathcal{C}_{1}=\left\{v \in W^{\Pi} ; S \circ v=0,\left(1-\epsilon_{\overline{0}}\right) \circ v=0\right\} \underset{\epsilon_{\overline{0}} \circ W=\widehat{W}}{=} \widehat{\mathcal{C}} \subset \widehat{W}^{\Pi} .
$$

Corollary 4.8. With the data from (108)-(112) any $\mathrm{z} \widehat{\mathrm{W}}$-behavior $\widehat{\mathcal{C}}$ gives rise to the A $W$-behavior $\mathcal{C}_{1}$, and indeed $\widehat{\mathcal{C}}=\mathcal{C}_{1}$ with the identification

$$
\mathbb{F}^{L}=\widehat{W}=\epsilon_{\overline{0}} \circ W=\left\{w \in W ; w=\epsilon_{\overline{0}} \circ w\right\} \subset W=\mathbb{F}^{N} .
$$

Corollary 4.9. For the ${ }_{\mathbf{A}} W$-behavior $\mathcal{B}$ and $\mathrm{z}_{\mathbf{z}} \widehat{W}$-behavior $\widehat{\mathcal{B}}$ from (107) Cor. 4.8 implies

$$
\mathcal{B} \cong \mathcal{B}_{1}=\widehat{\mathcal{B}}, w \mapsto w_{1}=\widehat{w}, w_{1}(\bar{j}, \lambda)(t)=\left\{\begin{array}{ll}
w(\lambda)(\sigma(\bar{j})+t) & \text { if } t \in L \\
0 & \text { if } t \in N \backslash L
\end{array} .\right.
$$

In particular, $\widehat{\mathcal{B}}$ is also canonically an ${ }_{\mathbf{A}} W$-behavior. On the module level the isomorphism (114) corresponds to the A-linear isomorphism

$$
\begin{aligned}
\mathbf{A}^{\bar{N} \times \Lambda} /\left(\mathbf{A}^{\bar{N} \times \Gamma} \widehat{R}+\mathbf{A}^{\bar{N} \times \Lambda} \epsilon_{0}\right) & \cong \\
\delta_{\bar{j}, \lambda}+\left(\mathbf{A}^{\bar{N} \times \Gamma} \widehat{R}+\mathbf{A}^{\bar{N} \times \Lambda} \epsilon_{0}\right) & \mapsto \epsilon_{\overline{0}} q^{\sigma(\bar{j})} \mathbf{w}_{\lambda} .
\end{aligned}
$$




\subsection{Applications}

Thms. 4.6 and 4.7 reduce the study of $\mathbf{A}_{\mathbf{A}} W$-behaviors to that of $\mathbf{z} \widehat{W}$-behaviors. Recall that every finitely generated (f.g.) A-module $M$ admits the $\mathbf{Z}$-decomposition

$$
M=\oplus_{\bar{i} \in \bar{N}} \epsilon_{\bar{i}} M=\oplus_{\bar{i} \in \bar{N}} q^{-\sigma(\bar{i})} \epsilon_{\overline{0}} q^{\sigma(\bar{i})} M=\oplus_{\bar{i} \in \bar{N}} q^{-\sigma(\bar{i})} \epsilon_{\overline{0}} M \cong\left(\epsilon_{\overline{0}} M\right)^{\overline{\mathbf{Z}}} .
$$

If a class $\mathfrak{C}$ of $\mathbf{Z}$-modules is closed under direct summands and finite direct sums then $M$ as $\mathbf{Z}$-module belongs to $\mathfrak{C}$ if and only if $\epsilon_{\overline{0}} M$ does. Many classes have this property. Here we give only some applications for multidimensional behaviors over a field $\mathbb{F}$. The field assumption implies that $\mathbf{Z}=\mathbb{F}[L]$ is a standard Laurent polynomial algebra, especially noetherian integral domain that has the standard quotient field $\mathbb{F}(L)=\mathbb{F}(x)$ of rational functions. The field assumption is made to enable the application of the quotient ring theory from [16]. An element $s \in \mathbf{A}$ is called regular if os : $\mathbf{A} \rightarrow$ $\mathbf{A}, a \mapsto a s$, and $s \circ: \mathbf{A} \rightarrow \mathbf{A}, a \mapsto s a$, are injective. The set $S:=S_{\mathbf{A}}$ of regular elements of $\mathbf{A}$ is a multiplicative submonoid $\mathbf{A}$. The commutative integral domain $\mathbf{Z}$ is noetherian and prime ( 0 is a prime ideal) and Morita equivalence preserves these properties [16, Prop. 3.5.10], hence $\mathbf{A}$ is noetherian and prime and therefore a (left and right) prime Goldie ring [16, §2.3.1]. By Goldie's theorem [16, Thm. 2.3.6] the set $S$ satisfies the left and right Ore condition [16, §2.1.6] and there exists the simple artinian quotient ring

$$
\mathbf{A} \subset \mathbf{Q}:=S^{-1} \mathbf{A}=\left\{\frac{a}{s}=s^{-1} a ; a \in \mathbf{A}, s \in S=S_{\mathbf{A}}\right\} .
$$

Likewise, every $M \in \mathbf{A}$ Mod gives rise to the quotient Q-module $S^{-1} M$ and the canonical map

$$
\begin{gathered}
\operatorname{can}_{M}: M \rightarrow S^{-1} M=\left\{\frac{x}{s}=s^{-1} x ; x \in M, s \in S\right\}, x \mapsto \frac{x}{1}, \text { with } \\
\operatorname{tor}_{\mathbf{A}}(M):=\operatorname{ker}\left(\operatorname{can}_{M}\right)=\{x \in M ; \exists s \in S \text { with } s x=0\} .
\end{gathered}
$$

The latter submodule is the (A-)torsion submodule of $M$ and $M$ is called A-torsion resp. torsionfree if $\operatorname{tor}_{\mathbf{A}}(M)=M$ resp. $\operatorname{tor}_{\mathbf{A}}(M)=0$. Obviously $\operatorname{tor}_{\mathbf{A}}(\mathbf{A})=0$. For the commutative integral domain $\mathbf{Z}$ and a $\mathbf{Z}$-module $P$ the corresponding data are

$$
S_{\mathbf{Z}}=\mathbf{Z} \backslash\{0\}, \mathbb{F}(L):=\operatorname{quot}(\mathbf{Z}):=S_{\mathbf{Z}}^{-1} \mathbf{Z}, S_{\mathbf{Z}}^{-1} P, \operatorname{tor}_{\mathbf{Z}}(P) .
$$

Lemma 4.10. (i) $S_{\mathbf{Z}}=\mathbf{Z} \cap S_{\mathbf{A}}$ (ii) $\operatorname{tor}_{\mathbf{A}}(M)=\operatorname{tor}_{\mathbf{Z}}(M) \cong \operatorname{tor}_{\mathbf{Z}}\left(\epsilon_{\overline{0}} M\right)^{\bar{N}}$.

Proof. (i) Recall $\epsilon_{\overline{0}} \mathbf{A} \cong \mathbf{Z}^{\bar{N}}$ from (78). If $s \in S_{\mathbf{Z}}$ then $\circ s: \mathbf{Z}^{\bar{N}} \rightarrow \mathbf{Z}^{\bar{N}}$ is injective and hence so are $\circ s: \epsilon_{\overline{0}} \mathbf{A} \rightarrow \epsilon_{\overline{0}} \mathbf{A}$ and, by Morita equivalence, $\circ s: \mathbf{A} \rightarrow \mathbf{A}$. Since $s$ belongs to the center $\mathbf{Z}$ of $\mathbf{A}$ this implies $S_{\mathbf{Z}} \subseteq \mathbf{Z} \cap S_{\mathbf{A}}$. The reverse inclusion is obvious.

(ii) For an A-module $M$ the isomorphism resp. inclusion

$$
\operatorname{tor}_{\mathbf{Z}}\left(\epsilon_{\overline{0}} M\right)^{\bar{N}} \cong \operatorname{tor}_{\mathbf{Z}}(M) \subseteq \operatorname{tor}_{\mathbf{A}}(M)
$$

follow from (116) resp. (i). The submodule $\operatorname{tor}_{\mathbf{Z}}(M)$ is an $\mathbf{A}$-submodule of $M$ and $M / \operatorname{tor}_{\mathbf{Z}}(M)$ is $\mathbf{Z}$-torsionfree. Therefore there is a $\mathbf{Z}$-monomorphism from this module into a free module and, due to $\epsilon_{\overline{0}} \mathbf{A} \cong \underset{\mathbf{Z}}{\cong} \mathbf{Z}^{\bar{N}}$, also a $\mathbf{Z}$-monomorphism 
$\epsilon_{\overline{0}}\left(M / \operatorname{tor}_{\mathbf{Z}}(M)\right) \rightarrow \epsilon_{\overline{0}} \mathbf{A}^{p}$. Morita equivalence implies an $\mathbf{A}$-monomorphism

$$
\begin{aligned}
M / \operatorname{tor}_{\mathbf{Z}}(M) \rightarrow & \mathbf{A}^{p} \Longrightarrow \operatorname{tor}_{\mathbf{A}}\left(M / \operatorname{tor}_{\mathbf{Z}}(M)\right)=0 \Longrightarrow \operatorname{tor}_{\mathbf{A}}(M) / \operatorname{tor}_{\mathbf{Z}}(M)=0 \Longrightarrow \\
& \operatorname{tor}_{\mathbf{A}}(M) \subseteq \operatorname{tor}_{\mathbf{Z}}(M) \Longrightarrow \operatorname{tor}_{\mathbf{A}}(M)=\operatorname{tor}_{\mathbf{Z}}(M) .
\end{aligned}
$$

Associated with a f.g. A-module $M$ are the module resp. behaviors (cf. Thm. 4.6)

$$
\epsilon_{\overline{0}} M \in \mathbf{z}_{\mathbf{z}} \operatorname{Mod} \text { resp. } \operatorname{Hom}_{\mathbf{A}}(M, W) \cong \mathcal{B} \cong \widehat{\mathcal{B}} \cong \operatorname{Hom}_{\mathbf{Z}}\left(\epsilon_{\overline{0}} M, \widehat{W}\right) .
$$

Recall that the $\mathbf{z} \widehat{W}$-behavior $\widehat{\mathcal{B}}$ is autonomous resp. controllable if $\epsilon_{\overline{0}} M$ is $\mathbf{Z}$-torsion resp. torsionfree. We use the same terminology for the isomorphic ${ }_{\mathbf{A}} W$-behavior $\mathcal{B}$.

Corollary 4.11. (i) The $\mathbf{A} W$-behavior $\mathcal{B} \cong \operatorname{Hom}_{\mathbf{A}}(M, W), W=\mathbb{F}^{N}$, is autonomous resp. controllable if and only if $\operatorname{tor}_{\mathbf{A}}(M)=M$ resp. $\operatorname{tor}_{\mathbf{A}}(M)=0$.

(ii) The behavior $\mathcal{B}$ is controllable if and only if there is a behavior surjection $W^{m} \rightarrow \mathcal{B}$ or, in Willems' language, an image representation of $\mathcal{B}$.

Proof. (i) follows directly from Lemma 4.10.

(ii) Note that $\operatorname{Hom}_{\mathbf{A}}(\mathbf{A}, W) \cong W$. By duality there is a behavior epimorphism $W^{m} \rightarrow$ $\mathcal{B}$ if and only if there is an $\mathbf{A}$-monomorphism $M \rightarrow \mathbf{A}^{m}$. The latter property implies $\operatorname{tor}_{\mathbf{A}}(M)=0$ and, equivalently, the controllability. If, conversely, $\operatorname{tor}_{\mathbf{A}}(M)=0$ then also tor $_{\mathbf{Z}}\left(\epsilon_{\overline{0}} M\right)=0$. As in part (ii) of the proof of Lemma 4.10 this implies an A-monomorphism $M \rightarrow \mathbf{A}^{m}$.

We finally consider input/output (IO) decompositions in the situation of Thm. 4.6 and 4.7. Such a decomposition for the behavior $\widehat{\mathcal{B}} \subseteq \widehat{W}^{\bar{N} \times \Lambda}$ is given by a disjoint decomposition

$$
\bar{N} \times \Lambda=\operatorname{inp} \uplus \text { out, } p:=\mid \text { out }|, m:=| \operatorname{inp}|, p+m=| \bar{N}|| \Lambda \mid,
$$

of the index set of the components $\widehat{w}_{\bar{j}, \lambda}$ of the trajectories of $\widehat{\mathcal{B}}$ with the following properties: We decompose $\widehat{R}$ and $\widehat{w}$ accordingly, i.e.,

$$
\begin{aligned}
& \widehat{R}=(\widehat{P},-\widehat{Q}) \in \mathbf{Z}^{(\bar{N} \times \Gamma) \times(\text { out } \uplus \text { inp })}, \widehat{w}=\left(\frac{\widehat{y}}{\widehat{u}}\right) \in \mathbb{F}^{\text {out } \uplus \text { inp }} \Longrightarrow \\
& \epsilon_{\overline{0}} M=\mathbf{Z}^{\text {out } \uplus \text { inp }} / \widehat{U}, \widehat{\mathcal{B}}=\left\{\left(\frac{\widehat{y}}{\widehat{u}}\right) \in \mathbb{F}^{\text {out } \uplus \text { inp }} ; \widehat{P} \circ \widehat{y}=\widehat{Q} \circ \widehat{u}\right\} .
\end{aligned}
$$

The defining properties of the $\mathrm{IO}$ decomposition are

$$
p=\operatorname{rank}(\widehat{U})=\operatorname{rank}(\widehat{R})=\operatorname{rank}(\widehat{P}) .
$$

The decomposition (121) with the property (123) is called an $I O$ decomposition and $\widehat{\mathcal{B}}$ with this structure an IO behavior. In general, the behavior admits several IO decompositions. The IO decompositions depend on $\widehat{U}$ or $\widehat{\mathcal{B}}$, but not on the special choice of $\widehat{R}$. The unique matrix $\widehat{H} \in \operatorname{quot}(\mathbf{Z})^{\text {out } \times \text { inp }}$ with $\widehat{P} \widehat{H}=\widehat{Q}$ does not depend on the special choice of $\widehat{R}$ and is called the transfer matrix of the IO behavior $\widehat{\mathcal{B}}$. The IO decomposition is also characterized by the properties that the projection

$$
\begin{aligned}
& \operatorname{proj}: \widehat{\mathcal{B}} \rightarrow \widehat{W}^{\text {inp }},\left(\begin{array}{l}
\widehat{y} \\
\widehat{u}
\end{array}\right) \mapsto \widehat{u}, \text { is surjective and } \\
& \widehat{\mathcal{B}}^{0}:=\left\{\widehat{y} \in \widehat{W}^{\text {out }} ; \widehat{P} \circ \widehat{y}=0\right\} \text { is autonomous }
\end{aligned}
$$


or, in module terms, that $\mathbf{Z}^{\text {out }} / \mathbf{Z}^{\bar{N} \times \Gamma} \widehat{P}$ is a torsion module and that

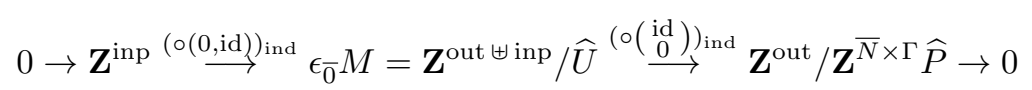

is exact. The surjectivity of proj : $\widehat{\mathcal{B}} \rightarrow \widehat{W}^{\text {inp }}$ and the autonomy of $\widehat{\mathcal{B}}^{0}$ justify the term input for $\widehat{u}$ : The components $\widehat{u}(\bar{j}, \lambda),(\bar{j}, \lambda) \in$ inp, can be chosen arbitrarily and $\widehat{u}$ is maximal with this property. In the language of Fliess the image $C:=$ $\operatorname{im}\left((\circ(0, \text { id }))_{\text {ind }}\right) \subseteq \epsilon_{\overline{0}} M$ is called a control submodule and the $u(\bar{j}, \lambda),(\bar{j}, \lambda) \in$ inp, are the associated controls.

Corollary 4.12. The ${ }_{\mathbf{A}} W$-behavior isomorphism $\mathcal{B} \cong \widehat{\mathcal{B}}$ from (107) and Cor. 4.9 and the IO structure from (121)-(125), viz.

$$
\begin{aligned}
\mathcal{B} \cong \mathcal{B}_{1}=\widehat{\mathcal{B}}, w \mapsto \widehat{w} & =\left(\begin{array}{ll}
\widehat{y} \\
\widehat{u}
\end{array}\right), \text { with } \\
\forall t \in L, \bar{j} \in \bar{N}, \lambda \in \Lambda: w(\lambda)(t+\sigma(\bar{j})) & = \begin{cases}y(\bar{j}, \lambda)(t) & \text { if }(\bar{j}, \Lambda) \in \text { out } \\
u(\bar{j}, \lambda)(t) & \text { if }(\bar{j}, \Lambda) \in \text { inp }\end{cases}
\end{aligned}
$$

imply that the components $w(\lambda) \mid(\sigma(\bar{j})+L), \quad(\bar{j}, \lambda) \in$ inp, of $w \in \mathcal{B}$ can be chosen arbitrarily and that these components are a maximal family with this property. In general, whole components $w(\lambda) \in \mathbb{F}^{N}$ cannot be chosen as input.

\subsection{Periodic trajectories}

We assume a commutative cogenerator ring $\mathbb{F}$ or, equivalently, that $\mathbb{F}$ is an injective cogenerator as module over itself. The assumptions (23)-(27) are in force. Additionally we introduce the signal module

$$
\begin{aligned}
& \bar{W}:=\mathbb{F}^{\bar{N}} \subset W=\mathbb{F}^{N} \text { and } \overline{\mathbf{A}}:=\mathbb{F}^{\bar{N}}[\bar{N} ; \circ]=\oplus_{\bar{j} \in \bar{N}} \mathbb{F}^{\bar{N}} q^{\bar{j}}=\oplus_{\bar{i}, \bar{j} \in \bar{N}} \mathbb{F} \epsilon_{\bar{i}} q^{\bar{j}} \text { with } \\
& \left(a q^{\bar{i}}\right)\left(b q^{\bar{j}}\right)=a(\bar{i} \circ b) q^{\bar{i}+\bar{j}}, a, b \in \mathbb{F}^{\bar{N}},(\bar{i} \circ b)(\bar{t}):=b(\bar{t}+\bar{i}), \bar{i}, \bar{j}, \bar{t} \in \bar{N}, \\
& \left(\left(a q^{\bar{i}}\right) \circ w\right)(\bar{t}):=a(\bar{t}) w(\bar{t}+\bar{i}), w \in \bar{W}=\mathbb{F}^{\bar{N}}, a \in \mathbb{F}^{\bar{N}}, \bar{i}, \bar{t} \in \bar{N} .
\end{aligned}
$$

The left ideal $\mathfrak{a}:=\sum_{j \in L} \mathbf{A}\left(q^{j}-1\right) \subset \mathbf{A}$ is indeed two-sided since $q^{j} \in \mathbf{Z}=$ center $(\mathbf{A})$ for $j \in L$ and the map

$$
\mathbf{A} / \mathfrak{a} \rightarrow \overline{\mathbf{A}}, a q^{j}+\mathfrak{a} \mapsto a q^{\bar{j}},
$$

is an $\mathbb{F}$-algebra isomorphism by which we identify $\mathbf{A} / \mathfrak{a}=\overline{\mathbf{A}}, q^{j}+\mathfrak{a}=q^{\bar{j}}$. Left $\overline{\mathbf{A}}$-modules $\bar{M}$ are the same as left $\mathbf{A}$-modules with $\mathfrak{a} \bar{M}=0$. Obviously

$$
\begin{gathered}
\bar{W}=\mathbb{F}^{\bar{N}}=\mathfrak{a}^{\perp}=\left\{w \in W=\mathbb{F}^{N} ; \forall i \in L: q^{i} \circ w=w\right\} \text { and } \\
\operatorname{Hom}_{\mathbf{A}}(\bar{M}, W)=\operatorname{Hom}_{\overline{\mathbf{A}}}(\bar{M}, \bar{W}) \ni \Phi \text { since } \mathfrak{a} \circ \Phi(\bar{M})=\Phi(\mathfrak{a} \bar{M})=\Phi(0)=0 .
\end{gathered}
$$

Corollary 4.13. (cf. [23]) The signal module $\overline{\mathrm{A}}_{\mathbf{A}} \bar{W}$ is an injective cogenerator. An ${ }_{\mathrm{A}} \bar{W}$-behavior $\overline{\mathcal{B}} \subseteq \bar{W}^{\Lambda}$ is the same as an ${ }_{\mathbf{A}} W$-behavior $\mathcal{B} \subseteq \bar{W}^{\Lambda}=\left(\mathfrak{a}^{\perp}\right)^{\Lambda} \subseteq W^{\Lambda}$, i.e., a $\mathcal{B}$ with L-periodic trajectories. Hence there is a categorical duality between f.g. left $\overline{\mathbf{A}}$-modules and $\mathbb{A}^{N} \mathbb{F}^{N}$-behaviors with L-periodic trajectories. 
Proof. This follows from (129) and the injective cogenerator property of ${ }_{\mathbf{A}} W$.

Corollary 4.14. If $\mathbb{F}$ is a cogenerator ring and $N$ a f.g. abelian group then $\mathbb{F}^{N}$ is an $\mathbb{F}^{N}[N ; \circ]$-cogenerator if and only if $N$ is finite. This proves (2) of Thm. 2.3.

Proof. This follows from $N \cong \mathbb{Z}^{m} \times \operatorname{tor}(N)$, Cor. 4.13 and (3) in Section 3.2.

Corollary 4.15. The $\overline{\mathbf{A}}$-module $\overline{\mathbf{A}} \epsilon_{\overline{0}}$ is a progenerator and Cor. 4.3 applies analogously, in particular,

$$
\overline{\mathbf{A}} \cong \operatorname{Hom}_{\mathbb{F}}\left(\overline{\mathbf{A}} \epsilon_{\overline{0}}, \overline{\mathbf{A}} \epsilon_{\overline{0}}\right) \cong \mathbb{F}^{\bar{N} \times \bar{N}} .
$$

The dual $\mathbb{F}$-module $\overline{\mathbf{A}}^{*}:=\operatorname{Hom}_{\mathbb{F}}(\overline{\mathbf{A}}, \mathbb{F})$ has the dual basis $\delta_{(\bar{i}, \bar{j})}$ of the $\mathbb{F}$-basis $\epsilon_{\bar{i}} \bar{q}^{\bar{j}}$ defined by

$$
\delta_{(\bar{i}, \bar{j})}\left(\epsilon_{\bar{k}} q^{\bar{\ell}}\right):=\delta_{\bar{i}, \bar{k}} \delta_{\bar{j}, \bar{\ell}} .
$$

Moreover $\overline{\mathbf{A}}^{*}$ is a left $\overline{\mathbf{A}}$-module via $(a \alpha)(b):=\alpha(b a)$ for $\alpha \in \overline{\mathbf{A}}^{*}$ and $a, b \in \overline{\mathbf{A}}$ and also a right $\overline{\mathbf{A}}$-module via $(\alpha a)(b)=\alpha(a b)$. Define

$$
\begin{gathered}
\omega \in \operatorname{Hom}_{\mathbb{F}}(\overline{\mathbf{A}}, \mathbb{F}) \text { by } \omega\left(\epsilon_{\bar{i}} q^{\bar{j}}\right)=\delta_{\overline{0}, \bar{j}} \Longrightarrow \\
\overline{\mathbf{A}} \Omega: \overline{\mathbf{A}} \rightarrow \overline{\mathbf{A}}^{*}, a \mapsto a \omega, \overline{\mathbf{A}} \Omega \in \operatorname{Hom}\left({ }_{\mathbf{A}} \mathbf{A}, \overline{\mathbf{A}} \mathbf{A}^{*}\right) .
\end{gathered}
$$

Likewise, $\Omega_{\overline{\mathbf{A}}}: \overline{\mathbf{A}} \rightarrow \overline{\mathbf{A}}^{*}, a \mapsto \omega a$, is right $\overline{\mathbf{A}}$-linear. We compute

$$
\begin{aligned}
& \overline{\mathbf{A}} \Omega\left(\epsilon_{\bar{i}} q^{\bar{j}}\right)\left(\epsilon_{\bar{k}} q^{\bar{\ell}}\right)=\omega\left(\epsilon_{\bar{k}} q^{\bar{\ell}} \epsilon_{\bar{i}} q^{\bar{j}}\right)=\omega\left(\epsilon_{\bar{k}} \epsilon_{\bar{i}-\bar{\ell}} q^{\bar{j}+\bar{\ell}}\right)= \\
& \delta_{\bar{k}, \bar{i}-\bar{\ell}} \delta_{\overline{0}, \bar{j}+\bar{\ell}}=\delta_{\bar{i}+\bar{j}, \bar{k}} \delta_{-\bar{j}, \bar{\ell}}=\delta_{(\bar{i}+\bar{j},-\bar{j})}\left(\epsilon_{\bar{k}} q^{\bar{\ell}}\right) \Longrightarrow \\
& \overline{\mathbf{A}} \Omega: \overline{\mathbf{A}} \overline{\mathbf{A}} \cong{ }_{\mathbf{A}} \overline{\mathbf{A}}^{*}={ }_{\overline{\mathbf{A}}} \operatorname{Hom}_{\mathbb{F}}(\overline{\mathbf{A}}, \mathbb{F}), \epsilon_{\bar{i}} q^{\bar{j}} \mapsto \delta_{(\bar{i}+\bar{j},-\bar{j})} \text {, and likewise } \\
& \Omega_{\overline{\mathbf{A}}}: \overline{\mathbf{A}}_{\overline{\mathbf{A}}} \cong \overline{\mathbf{A}}_{\overline{\mathbf{A}}}^{*} \text {. }
\end{aligned}
$$

Since ${ }_{\mathbb{F}} \mathbb{F}$ is an injective cogenerator the dual module $\operatorname{Hom}_{\mathbb{F}}(\overline{\mathbf{A}}, \mathbb{F})$ is an injective $\overline{\mathbf{A}}$ cogenerator. This standard result is shown as in (20) with $\overline{\mathbf{A}}$ instead of $\mathbb{F}[N]$.

Corollary 4.16. The $\mathbb{F}$-algebra $\overline{\mathbf{A}}$ is injective and a cogenerator both as left and right $\overline{\mathbf{A}}$-module and therefore a (noncommutative) cogenerator ring $[14, \S 19 B]$. If $\mathbb{F}$ is additionally artinian and thus a commutative quasi-Frobenius ring then so is $\overline{\mathbf{A}}$ as f.g. $\mathbb{F}$-module [14, Thm. 15.1].

Proof. The isomorphisms $\overline{\mathbf{A}} \cong \overline{\mathbf{A}}^{*}$ and the preceding remark imply that $\mathbf{A}$ is a left and right $\overline{\mathbf{A}}$-injective cogenerator.

\section{$5 \quad$ Relation to previous work}

We describe the connection of the method of Section 4 with the important previous work for one-dimensional discrete LTV-behaviors with periodic coefficients in a field from, for instance, [12], [1] and [2]. The assumptions are those of Section 4:

$$
\begin{aligned}
& \text { a quasi-Frobenius ring } \mathbb{F}, N:=\mathbb{Z}^{r}, \mathbb{Z}^{r} \cong L \subset N, \bar{N}:=N / L, W=\mathbb{F}^{N}, \\
& \begin{array}{c}
\sigma: \bar{N} \rightarrow N, \overline{\sigma(\bar{j})}=\sigma(\bar{j})+L=\bar{j} \in \bar{N}, L \times \bar{N} \cong N,(\tau, \bar{j}) \mapsto \tau+\sigma(\bar{j}), \\
\mathbf{Z}=\mathbb{F}[L] \subset \mathbb{F}[N] \subset \mathbf{A}=\mathbb{F}^{N}[N ; \circ] .
\end{array}
\end{aligned}
$$


The elements of $N$ resp. $L$ are generically denoted by $t$ resp. by $\tau$. The quoted papers use $\mathbb{F}=\mathbb{R}$ or $\mathbb{F}=\mathbb{C}$ and $N=\mathbb{Z} \supset L=\mathbb{Z} n$. We transfer the definitions of the quoted papers to the more general situation here.

Since $\mathbf{A}$ and $\mathbb{F}[N]$ act on $\mathbb{F}^{N}$ so does $\mathbf{Z}=\mathbb{F}[L]$. The isomorphism

$$
\mathbb{F}^{N} \cong\left(\mathbb{F}^{L}\right)^{\bar{N}}=\mathbb{F}^{L \times \bar{N}}, w \mapsto \widehat{w}, w(\tau+\sigma(\bar{j}))=\widehat{w}(\bar{j})(\tau)=\widehat{w}(\tau, \bar{j}),
$$

is $\mathbb{F}[L]$-linear. Since $\mathbb{F}[L] \mathbb{F}^{L}$ is an injective cogenerator so is ${ }_{\mathbb{F}[L]} \mathbb{F}^{N}$. Recall the $\mathbb{F}[L]$ decomposition

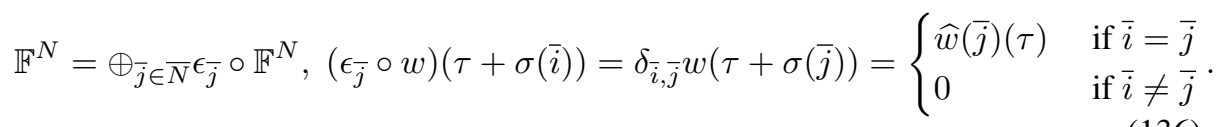

Thus we may and do identify

$$
\mathbb{F}^{N}=\left(\mathbb{F}^{L}\right)^{\bar{N}} \supset \epsilon_{\bar{j}} \circ \mathbb{F}^{N}=0 \times \cdots \times 0 \times \bar{F}^{\bar{j}} \times 0 \times \cdots \times 0 \ni \epsilon_{\bar{j}} \circ w=\widehat{w}(\bar{j}) .
$$

For any finite index set $\Lambda$ the preceding identification implies

$$
\left(\mathbb{F}^{N}\right)^{\Lambda}=\left(\mathbb{F}^{L}\right)^{\bar{N} \times \Lambda} \supset \epsilon_{\bar{j}} \circ\left(\mathbb{F}^{N}\right)^{\Lambda}=0 \times \cdots \times 0 \times\left(\mathbb{F}^{\bar{j}}\right)^{\Lambda} \times 0 \times \cdots \times 0 .
$$

In this fashion the $\mathbb{F}[L]$-isomorphisms

$$
W^{\Lambda}=\left(\mathbb{F}^{\bar{N}}\right)^{\Lambda}=\mathbb{F}^{\bar{N} \times \Lambda} \cong \mathbb{F}^{L \times \bar{N} \times \Lambda}=\left(\mathbb{F}^{L}\right)^{\bar{N} \times \Lambda}=\widehat{W}^{\bar{N} \times \Lambda}, \mathcal{B} \cong \widehat{\mathcal{B}},
$$

from (101) become the identities

$$
W^{\Lambda}=\widehat{W}^{\bar{N} \times \Lambda} \supset \mathcal{B}=\widehat{\mathcal{B}} .
$$

Definition 5.1. 1. (cf. $[12$, Thm. 8],[2, §3]) An $L$-periodic behavior is an $\mathbb{F}[L]$ subbehavior $\widehat{\mathcal{B}}$ of some $W^{\Lambda}=\left(\mathbb{F}^{N}\right)^{\Lambda}=\left(\mathbb{F}^{L}\right)^{\bar{N} \times \Lambda}=\widehat{W}^{\bar{N} \times \Lambda}$.

2. If $\mathbb{F}$ is a field this is the same as a closed $\mathbb{F}$-subspace of $\widehat{\mathcal{B}} \subseteq \mathbb{F}^{N \times \Lambda}$ that is invariant under the action of $L$ on $\mathbb{F}^{N}$.

3. If $\mathbb{F}$ is a field, $N=\mathbb{Z} \supset L=\mathbb{Z} n$ and $\widehat{\mathcal{B}} \subset \widehat{W^{N} \times \Lambda}$ is a closed subspace the invariance means $q^{n} \circ \widehat{\mathcal{B}}=\widehat{\mathcal{B}}$. This is the case treated in [12], [2].

We use the identifications (140) and (95), i.e.,

$$
\mathbf{Z}^{\bar{N} \times \Lambda}=\epsilon_{\overline{0}} \mathbf{A}^{\Lambda}=\oplus_{\bar{j} \in \bar{N}} \mathbf{Z} \epsilon_{\overline{0}} q^{\sigma(\bar{j})} \delta_{\lambda} \ni \delta_{(\bar{j}, \lambda)}=\epsilon_{\overline{0}} q^{\sigma(\bar{j})} \delta_{\lambda} .
$$

The $\mathbb{F}[L]$-subbehavior $\widehat{\mathcal{B}} \subseteq \widehat{W}^{\bar{N} \times \Lambda}$ has the unique form

$$
\begin{gathered}
\widehat{\mathcal{B}}=\widehat{U}^{\perp}, \widehat{U} \subseteq_{\mathbb{F}[L]} \mathbb{F}[L]^{\bar{N} \times \Lambda}=\epsilon_{\overline{0}} \mathbf{A}^{\Lambda} \Longrightarrow \text { exists uniquely } \\
U \subseteq \subseteq_{\mathbf{A}} \mathbf{A}^{\Lambda} \text { with } \epsilon_{\overline{0}} U=\widehat{U}, \widehat{\mathcal{B}} \underset{(140)}{=\mathcal{B}}:=U^{\perp} \subseteq W_{(140)}^{\Lambda} \underset{W^{\bar{N}} \times \Lambda}{ } .
\end{gathered}
$$


Corollary 5.2. (cf. [12, Thm. 8], [2, §3]) An L-periodic behavior $\widehat{\mathcal{B}} \subseteq W^{\Lambda}=\widehat{W}^{\bar{N} \times \Lambda}$ in the sense of Def. 5.1 is the same as an $\mathrm{A} W$-subbehavior of $W^{\Lambda}$ and thus uniquely of the form $\mathcal{B}=U^{\perp}$ where $U \subseteq \mathbf{A}^{\Lambda}$. If $U=\mathbf{A}^{\Gamma} R$ for some matrix $R \in \mathbf{A}^{\Gamma \times \Lambda}$ then

$$
\mathcal{B}=\left\{w \in W^{\Lambda} ; R \circ w=0\right\}, R \in \mathbf{A}^{\Gamma \times \Lambda} .
$$

The matrix $R$ has the unique representation

$$
\begin{aligned}
R=\sum_{\bar{j} \in \bar{N}} \epsilon_{\bar{j}} R_{j}, R_{j} \in \mathbb{F}[N] & =\oplus_{i \in N} \mathbb{F} q^{i} \Longrightarrow(R \circ w)(t)=\sum_{\bar{j} \in \bar{N}} \delta_{\bar{t}, \bar{j}}\left(R_{j} \circ w\right)(t) \text { with } \\
\delta_{\bar{t}, \bar{j}}\left(R_{j} \circ w\right)(t) & = \begin{cases}\left(R_{j} \circ w\right)(t) & \text { if } \bar{t}=\bar{j} \text { or } t \in \sigma(\bar{j})+L \\
0 & \text { if } \bar{t} \neq \bar{j} \text { or } t \notin \sigma(\bar{j})+L\end{cases}
\end{aligned}
$$

\section{References}

[1] J. C. Aleixo, P. Rocha, and J.W. Polderman, 'A Contribution to the Study of Periodic Systems in the Behavioral Approach', pp.23-38 in Taming Heterogeneity and Complexity in Embedded Control, F. Lamnabhi-Laguarrigue, A. Loria, E. Planteley and S. Lagarouche, Eds., ISTE/Hermes Science Publications, Paris, 2007

[2] J. Aleixo, J.W. Polderman, P. Rocha, 'Representations and structural properties of periodic systems', Automatica 43(2007), 1921-1931

[3] S. Bittanti, P. Colaneri, Periodic Systems: Filtering and Control, Springer, 2009

[4] H. Bourlès, B. Marinescu, Linear Time-Varying Systems, Springer, Berlin, 2011

[5] H. Bourlès, B. Marinescu, U. Oberst, 'Exponentially stable linear time-varying discrete behaviors', submitted in revised form to SIAM J. Control Optim., 2015

[6] H. Bourlès, U. Oberst, 'Generalized convolution behaviors and topological algebra', pp. 1-42, Acta Appl Math 2015, DOI 10.1007/s10440-015-0007-4

[7] F. Chyzak, A. Quadrat, D. Robertz, 'OreModules: A symbolic package for the study of multidimensional linear systems' in J. Chiasson, J.-J. Loiseau (Eds.), Applications of Time-Delay Systems, Lecture Notes in Control and Information Sciences 352, Springer, 2007, pp. 233-264.

[8] M. Fliess, 'Some basic structural properties of generalized linear systems', Systems and Control Letters 15(1990), 391-396

[9] S. Fröhler, U. Oberst, 'Continuous time-varying linear systems', Systems and Control Letters 35(1998), 97-110

[10] H. Glüsing-Lürssen, P. Vettori, S. Zampieri, 'The algebraic structure of DD systems: a behavioral perspective', Kybernetica 37(2001), 397-426

[11] M. Kashiwara, T. Kawai, T. Kimura, Foundations of Algebraic Analysis, Princeton University Press, 1986 
[12] M. Kuijper, J.C. Willems, 'A behavioral framework for periodically time-varying systems', Proc. 36th IEEE Conference on Decision and Control, San Diego, 1997

[13] M. Kuijper, R. Pinto, J.W. Polderman, P. Rocha, 'Autonomicity and the absence of free variables for behaviors over finite rings', Proc. 7th Portuguese Conference on Automatic Control', Lisbon, Portugal, 2006

[14] T.Y. Lam, Lectures on Modules and Rings, Springer, New York, 1999

[15] P. Lu, M. Liu, U. Oberst, 'Linear Recurring Arrays, Linear Systems and Multidimensional Cyclic Codes over Quasi-Frobenius Rings', Acta Appl. Math. 80(2004), 175-198

[16] J.C. McConnell, J.C. Robson, Noncommutative Noetherian Rings, John Wiley and Sons, Chichester, 1987

[17] A.A. Nechaev, 'Linear recurrence sequences over commutative rings', Discrete Math. Appl. 2(1992), 659-683

[18] U. Oberst, 'Multidimensional Constant Linear Systems', Acta Appl. Math. 20(1990), 1-175

[19] A. Quadrat, Algebraic Analysis of Linear Functional Systems, book in preparation, 2015

[20] D. Robertz, 'Recent Progress in an Algebraic Analysis Approach to Linear Systems', Mult Syst Sign Process 2015, DOI 10.1007/s11045-014-0280-9

[21] W.J. Rugh, Linear System Theory, Prentice Hall, Upper Saddle River, NJ, 1996

[22] E. Zerz, "An Algebraic Analysis Approach to Linear Time-varying Systems', IMA J. Math. Control Inform. 23(2006), 113-126

[23] E. Zerz, 'On periodic solutions of linear difference equations', Proceedings MTNS 2010, 1567-1570, Budapest, 2010

[24] E. Zerz, L. Wagner 'Finite Multidimensional Behaviors', Multidimensional Systems and Signal Processing 23(2012), 5-15 